\title{
Risk Horizon and Rebalancing Horizon in Portfolio Risk Measurement
}

\author{
Paul Glasserman* \\ Columbia Business School
}

April 2009; revised December 2009

\begin{abstract}
This paper analyzes portfolio risk and volatility in the presence of constraints on portfolio rebalancing frequency. This investigation is motivated by the incremental risk charge (IRC) introduced by the Basel Committee on Banking Supervision. In contrast to the standard market risk measure based on a ten-day value-at-risk calculated at $99 \%$ confidence, the IRC considers more extreme losses and is measured over a one-year horizon. More importantly, whereas ten-day VaR is ordinarily calculated with a portfolio's holdings held fixed, the IRC assumes a portfolio is managed dynamically to a target level of risk, with constraints on rebalancing frequency. The IRC uses discrete rebalancing intervals (e.g., monthly or quarterly) as a rough measure of potential illiquidity in underlying assets. We analyze the effect of these rebalancing intervals on the portfolio's profit and loss distribution over a risk-measurement horizon. We derive limiting results, as the rebalancing frequency increases, for the difference between discretely and continuously rebalanced portfolios; we use these to approximate the loss distribution for the discretely rebalanced portfolio relative to the continuously rebalanced portfolio. Our analysis leads to explicit measures of the impact of discrete rebalancing under a simple model of asset dynamics.
\end{abstract}

\section{Introduction}

In 2007, the Basel Committee on Banking Supervision proposed a new measure of market risk known as the incremental risk charge, or IRC; the proposal was updated in January 2009 and is expected to take effect in 2010. The IRC is incremental to the current Basel standard for market risk, which is based on a $99 \%$ value-at-risk (VaR) measured over a ten-day horizon. The standard VaR measure was designed to capture the risk in short-term fluctuations of highly liquid securities; but, over time, banks have come to include less liquid assets in their trading books. The IRC arose in response to this changing composition of bank portfolios and as part of a broader attempt to equalize capital charges for similar risks held in banking and trading books. The proposal is detailed in reports of the Basel Committee $([4,5])$; for perspectives from the industry, see Duncan et al. [17], Sebton et al. [33], and Smillie and Epperlein [34]. For general background on risk capital, see, e.g., Crouhy, Galai, and Mark [11] and McNeil, Frey, and Embrechts [30].

*403 Uris Hall, Columbia Business School, New York, NY 10027, pg20@columbia.edu 
The Basel principles for calculating the IRC include the following key elements:

○ a $99.9 \% \mathrm{VaR}$

- measured over a one-year time horizon,

- assuming the portfolio is managed to a target level or risk,

○ incorporating "liquidity horizons" for less liquid assets.

The first two features are stringent but otherwise straightforward extensions of the traditional tenday $99 \% \mathrm{VaR}$; the last two features are novel. A standard ten-day VaR calculation uses a "buy and hold" assumption under which a portfolio is held fixed throughout the VaR horizon while market prices change. The IRC recognizes the implausibility of this assumption over a one-year horizon and allows the portfolio composition to change within the VaR interval. The notion of a target level of risk precludes a calculation in which a portfolio is artificially assumed to move to safe assets following losses; instead, the portfolio must be rebalanced to reflect a level of risk representative of the bank's trading. The last feature of the IRC limits the frequency of rebalancing to capture potential illiquidity in the underlying assets. A typical rebalancing frequency is monthly or quarterly.

The purpose of this article is to analyze these features of the proposed risk measure, with particular emphasis on the impact of the liquidity intervals nested within the longer risk horizon. The IRC provides the motivation for this investigation, but we see these features of the IRC as having broader applicability - any prescription for measuring portfolio risk over a moderately long horizon must address the question of how the portfolio evolves over the horizon; a target level of risk with constraints on rebalancing frequency provides a natural framework. Moreover, guidelines put forward by the Basel Committee often influence risk measurement and risk management even beyond the domain of bank supervision.

We analyze the impact of rebalancing frequency in a simple model in which the underlying assets evolve as correlated geometric Brownian motions. We model the target level of risk through a fixed level of portfolio volatility achieved through a fixed set of portfolio weights. We then analyze the difference between a continuously rebalanced portfolio and a portfolio with the same target weights that is rebalanced only periodically. This is a stylized model that does not capture effects like downgrades or defaults, but it leads to explicit characterizations of the impact of discrete rebalancing.

Our analysis is based on letting the rebalancing frequency increase, through which we derive several results. We establish a central limit theorem for the relative difference between the two portfolio distributions at a fixed horizon (the risk horizon of, e.g., one year) and a corresponding 
limit for the absolute difference as a mixture of normals. We show that the relative error is asymptotically independent of the level of the continuously rebalanced portfolio. We then use this result to derive an asymptotic "volatility adjustment" to correct for the effect of discrete rebalancing; somewhat surprisingly, this correction involves the asymptotic covariance between the relative error and the (log of the) continuous portfolio, despite their asymptotic independence. Next, we examine the relation between the discrete and continuous portfolios in the extreme tails, motivated by the $99.9 \%$ confidence level specified in the IRC. We show that, conditional on a large loss in the continuously rebalanced portfolio, the distribution of the discretely rebalanced portfolio is asymptotically concentrated at a point. We use this to derive an approximation to the extreme VaR and combine it with the volatility adjustment derived through the central limit theorem. Our approximations to the loss distribution are applicable to expected shortfall and other tail risk measures, in addition to VaR. Most of our results are formulated in a setting that contrasts fully continuous rebalancing with fully discrete rebalancing at fixed intervals, but we also discuss extensions to settings in which different subportfolios are rebalanced at different frequencies.

Discretely rebalanced portfolios arise in models of transaction costs and discrete hedging, including Bertsimas, Kogan, and Lo [6], Boyle and Emanuel [8], Duffie and Sun [15], Leland [29], and Morton and Pliska [31]. Gordy and Howells [19] compare the effect of alternative rebalancing assumptions on the procyclicality of capital requirements. Guasoni, Huberman, and Wang [20] analyze the effect of discrete rebalancing on the measurement of tracking error and portfolio alpha. Dufresne [16] compares the distributions of discretely and continuously weighted averages of asset prices. The distribution of the difference between a diffusion process and its discrete-time approximation has also received extensive study in the simulation literature, as in Kurtz and Protter [27]. We will comment further on connections and differences between our analysis and earlier work after presenting our first main result. As discussed above, the rebalancing constraints in the IRC serve as a rough way to capture potential illiquidity in measuring portfolio risk. Bangia, Diebold, Schuermann and Stroughair [3], and Jorion [25] calculate liquidity-adjusted VaR using information on bid-ask spreads. The two approaches could potentially be aligned by using a measure of market impact to set rebalancing frequencies, along the lines in Dubil [14].

Our analysis considers only the impact of rebalancing frequency and does not address other aspects of the IRC and the effect of illiquidity. For example, one concern in discussions of the IRC is "double counting" of risk when a bank calculates both a specific risk charge and the IRC. Other points of discussion include the justification for adding a one-year VaR number to a standard ten-day VaR, as well as the limitations of VaR more generally; see Finger [18] for an overview and commentary. We also stress that our analysis is limited to a simple model of asset dynamics and portfolio rebalancing. The benefit of working with a simple model is that it leads to explicit results 
that may provide a useful benchmark and suggest directions for further investigation.

The rest of the paper is organized as follows. Section 2 describes the modeling framework for our investigation and introduces notation. Section 3 establishes the central limit theorem for the relative difference between the discrete and continuous portfolios and derives an explicit expression for the limiting variance parameter in terms of underlying asset volatilities, correlations, and portfolio weights. Section 4 uses this to derive our adjustment to portfolio volatility — an adjustment that corrects the volatility for discrete rebalancing. Section 5 proves a conditional limit theorem for the loss in the discrete portfolio conditional on a large loss in the continuous portfolio. This section also reports numerical results to evaluate the quality of various approximations to the tail of the loss distribution. Section 6 considers the extension to subportfolios rebalanced at different frequencies, and Section 7 concludes the paper.

\section{Model Dynamics}

We begin by describing the evolution of the assets in the portfolios we consider. A $d$-dimensional standard Brownian motion $W=\left(W_{1}, \ldots, W_{d}\right)^{\top}$ drives the prices of $d$ assets with dynamics

$$
\frac{d S_{i}(t)}{S_{i}(t)}=\mu_{i} d t+\sum_{j=1}^{d} \sigma_{i j} d W_{j}(t), \quad i=1, \ldots, d .
$$

The drifts $\mu_{i}$ and volatility coefficients $\sigma_{i j}$ are constants. The covariance matrix $\Sigma$ of the instantaneous returns has entries

$$
\Sigma_{i j}=\sum_{k=1}^{d} \sigma_{i k} \sigma_{j k}, \quad i, j=1, \ldots, d .
$$

Given these asset dynamics, the portfolio is described by a fixed vector $w=\left(w_{1}, \ldots, w_{d}\right)^{\top}$ of weights. The weights sum to 1 , but some could be negative, reflecting short positions. The weights could be the result of a portfolio optimization problem, but we will simply take them as given. In the absence of liquidity constraints, the portfolio is continuously rebalanced to maintain the portfolio weights, so the value $V$ evolves as

$$
\frac{d V(t)}{V(t)}=\sum_{i=1}^{d} w_{i} \frac{d S_{i}(t)}{S_{i}(t)}
$$

which we write as

$$
\frac{d V(t)}{V(t)}=\mu_{w} d t+\sum_{i=1}^{d} w_{i} \sigma_{i}^{\top} d W(t),
$$

by defining $\mu_{w}=\sum_{i} w_{i} \mu_{i}$ and letting $\sigma_{i}=\left(\sigma_{i 1}, \ldots, \sigma_{i d}\right)^{\top}$. The portfolio's volatility is

$$
\sigma_{w}=\sqrt{w^{\top} \Sigma w}
$$


Indeed, we could write the dynamics of the continuously rebalanced portfolio as

$$
\frac{d V(t)}{V(t)}=\mu_{w} d t+\sigma_{w} d \tilde{W}(t)
$$

by introducing the scalar Brownian motion $\tilde{W}(t)=\sum_{i, j} w_{i} \sigma_{i j} W_{j}(t) / \sigma_{w}$. If we define

$$
\bar{\sigma}_{j}=\sum_{i=1}^{d} w_{i} \sigma_{i j}, \quad j=1, \ldots, d,
$$

then we also have $\sigma_{w}=\|\bar{\sigma}\|$.

Now introduce a risk horizon $T$ (e.g., 1 year) and a liquidity or rebalancing horizon $\Delta t=T / N$ (e.g., 1 month with $N=12$ ). The discretely rebalanced portfolio $\hat{V}$ evolves from $n \Delta t$ to $(n+1) \Delta t$ according to

$$
\hat{V}((n+1) \Delta t)=\hat{V}(n \Delta t)\left(\sum_{i=1}^{d} w_{i} \frac{S_{i}((n+1) \Delta t)}{S_{i}(n \Delta t)}\right) .
$$

In other words, the discretely rebalanced portfolio grows according to the weighted average of the holding period returns of the individual assets. We adopt the normalization $V(0)=\hat{V}(0)=1$.

Write $\hat{V}_{n}=\hat{V}(n \Delta t), V_{n}=V(n \Delta t)$, and $\Delta W(n)=W((n+1) \Delta t)-W(n \Delta t)$. The following easily verified representation of the difference between the two portfolio values will be useful:

$$
\hat{V}_{N}-V_{N}=V_{N} \sum_{n=1}^{N}\left(\frac{\hat{V}_{n}}{V_{n}}-\frac{\hat{V}_{n-1}}{V_{n-1}}\right) .
$$

The key to our analysis will be approximating the increments in (2) using, for $n=1, \ldots, N$,

$$
\epsilon_{n}=\frac{1}{2}\left(\sum_{i=1}^{d} w_{i}\left(\sigma_{i}^{\top} \Delta W(n)\right)^{2}-\left(\sum_{i=1}^{d} w_{i} \sigma_{i}^{\top} \Delta W(n)\right)^{2}\right)-\theta \Delta t,
$$

where

$$
\theta=\frac{1}{2}\left(\sum_{i=1}^{d} w_{i}\left\|\sigma_{i}\right\|^{2}-\sigma_{w}^{2}\right)=\frac{1}{2}\left(\sum_{i=1}^{d} w_{i}\left\|\sigma_{i}\right\|^{2}-\|\bar{\sigma}\|^{2}\right),
$$

recalling (1). These terms satisfy $E\left[\epsilon_{n}\right]=0$. In fact,

$$
E\left[\left(\sigma_{i}^{\top} \Delta W(n)\right)^{2}\right]=\left\|\sigma_{i}\right\|^{2} \Delta t \quad \text { and } \quad E\left[\left(\sum_{i=1}^{d} w_{i} \sigma_{i}^{\top} \Delta W(n)\right)^{2}\right]=\sigma_{w}^{2} \Delta t,
$$

so $\epsilon_{n}$ may be expressed as the difference of two terms, each having mean zero.

Our investigation focuses on the simple model of asset dynamics and portfolio weights described above. In practice, both the portfolio strategy and asset dynamics are likely to be more complex. The benefit of working with a simple model is that it leads to explicit results that can provide a useful benchmark for evaluating the effect of rebalancing frequency. For risk management purposes, the objective is not necessarily to reproduce a detailed trading strategy but, in the case of the IRC, to capture the notion of a target level of risk. In our setting, that target is captured by $\sigma_{w}$. 


\section{Asymptotic Error}

In this section, we analyze the difference $\hat{V}_{N}-V_{N}$ between the discretely rebalanced and continuously rebalanced portfolios as the number of rebalancing dates $N$ grows, with the risk horizon $T$ held fixed. We identify the limiting distributions of the absolute and relative errors. Our analysis builds on the representation in (2). The first step is to approximate the sum in (2) with the sum of the $\epsilon_{n}$ defined in (3). The proof of the following result (and most others) is deferred to the appendix.

\section{Proposition 1}

$$
E\left[\left(\frac{\hat{V}_{N}-V_{N}}{V_{N}}-\sum_{n=1}^{N} \epsilon_{n}\right)^{2}\right]=O\left(\Delta t^{2}\right) .
$$

This result underlies most of our analysis: it states that the relative difference between the discretely and continuously rebalanced portfolio values is nearly equal to a sum of independent errors defined through the squares of the Brownian increments driving the underlying asset returns.

\subsection{The Limit Theorem}

We now come to the main result of this section. To state it, we set

$$
\sigma_{L}^{2}=\operatorname{Var}\left[\frac{1}{2}\left(\sum_{i=1}^{d} w_{i}\left(\sigma_{i}^{\top} Z\right)^{2}-\left(\sum_{i=1}^{d} w_{i} \sigma_{i}^{\top} Z\right)^{2}\right)\right]
$$

with $Z \sim N(0, I)$ in $\mathbb{R}^{d}$. From the definition of $\epsilon_{n}$ in (3), we find that $\operatorname{Var}\left[\epsilon_{n}\right]=\sigma_{L}^{2} \Delta t^{2}$.

Theorem 1 As $N \rightarrow \infty$,

$$
\sqrt{N}\left(\hat{V}_{N}-V(T), \frac{\hat{V}_{N}-V(T)}{V(T)}\right) \Rightarrow(V(T) X, X),
$$

where $X \sim N\left(0, \sigma_{L}^{2} T^{2}\right)$ is independent of $V(T)$, and " $\Rightarrow$ " denotes convergence in distribution.

Proof. For each $N$, the $\epsilon_{n}, n=1, \ldots, N$, are i.i.d. with mean zero and variance $\sigma_{L}^{2} \Delta t^{2}$. Also, $E\left|\epsilon_{n}\right|^{3}=O\left(\Delta t^{3}\right)$. Thus, by the Lyapunov central limit theorem (as in, e.g., Theorem 7.1.2 of Chung [10]),

$$
\sqrt{N} \sum_{n=1}^{N} \epsilon_{n} \Rightarrow N\left(0, \sigma_{L}^{2} T^{2}\right)
$$

Let

$$
X_{N}=\sqrt{N}\left(\frac{\hat{V}_{N}-V(T)}{V(T)}\right)
$$

then, by Proposition $1, X_{N}-\sqrt{N} \sum_{n=1}^{N} \epsilon_{n} \Rightarrow 0$, and, by Theorem 4.4.6 of Chung [10], $X_{N}$ inherits the limiting distribution in (8). 
For any $j, k, m=1, \ldots, d, E\left[\Delta W_{j}(n) \Delta W_{k}(n) \Delta W_{m}(n)\right]=0$; it then follows from the fact that $\epsilon_{n}$ is a quadratic function of the components of $\Delta W(n)$ that $E\left[\epsilon_{n} \Delta W_{m}(n)\right]=0, m=1, \ldots, d$. In other words, $\epsilon_{n}$ is uncorrelated with the components of $\Delta W(n)$. The pairs $\left(\Delta W(n), \epsilon_{n}\right), n=1,2, \ldots, N$, are i.i.d. in $\mathbb{R}^{d+1}$, so the convergence in (8) extends to

$$
\frac{1}{\sqrt{N}}\left(\sum_{n=1}^{N} \frac{\Delta W(n)}{\sqrt{\Delta t}}, \sum_{n=1}^{N} \frac{\epsilon_{n}}{\sigma_{L} \Delta t}\right) \Rightarrow N(0, I)
$$

in $\mathbb{R}^{d+1}$. But then, by the Cramér-Wold device (Billingsley [7], p.49), $\left(W(T), X_{N}\right)$ is also asymptotically multivariate normal with independent components. Because $V(T)$ is a continuous function of $W(T)$, we also have $\left(V(T), X_{N}\right) \Rightarrow(V(T), X)$, with asymptotic independence, from which we get the convergence of $\left(V(T) X_{N}, X_{N}\right)$ in the statement of the theorem.

The convergence in (10) can be strengthened to a functional central limit theorem,

$$
\left\{\left(\sum_{n=1}^{[N t]} \Delta W(n), \sum_{n=1}^{[N t]} \frac{\epsilon_{n}}{\sigma_{L} \sqrt{\Delta t}}\right), t \in[0, T]\right\} \Rightarrow\{(W(t), \tilde{W}(t)), t \in[0, T]\}
$$

where $\tilde{W}$ is a one-dimensional Brownian motion independent of $W,[N t]$ denotes the integer part of $N t$ (see Billingsley [7], p.68). We may also write (7) as

$$
\sqrt{N}\left(\hat{V}_{N}-V(T), \frac{\hat{V}_{N}-V(T)}{V(T)}\right) \Rightarrow\left(V(T) \sigma_{L} \sqrt{T} \tilde{W}(T), \sigma_{L} \sqrt{T} \tilde{W}(T)\right),
$$

with $\tilde{W}$ a Brownian motion independent of $V$. This says neither more nor less than (7) - any normal random variable may be represented as the level of a Brownian motion at a fixed time but it is suggestive of the following interpretation: asymptotically, the rebalancing errors are driven by a Brownian motion that is independent of the Brownian motion driving the underlying assets.

Related results arise in other contexts. Kurtz and Protter [27] identify the law of the error of the Euler scheme for discretizing stochastic differential equations, and they characterize the limiting error through a stochastic differential equation driven by a Brownian motion independent of the original driving Brownian motion. Jacod and Protter [24] extend this analysis to Lévy-driven processes, and Detemple, Garcia, and Rindisbacher [12] use it to compare alternative discretization methods. Related ideas arise in the convergence of GARCH models to continuous-time limits, as in Duan [13]. Boyle and Emanuel [8] and, in a more general setting, Bertsimas, Kogan, and Lo [6], analyze the hedging error that results from applying a continuous-time delta-hedging strategy at discrete dates. Boyle and Emanuel [8] show that the hedging error is uncorrelated with the return on the underlying stock over a single period; Bertsimas et al. [6] find the limiting distribution of the hedging error over multiple periods and note that the hedging error is asymptotically independent 
of the price of the underlying asset. Related results are derived by Leland [29] in the context of option pricing with transaction costs.

A feature of our setting is that it allows an explicit representation of the limiting variance parameter $\sigma_{L}^{2}$. For this, we write (3) as

$$
\begin{aligned}
\epsilon_{n} & =\frac{1}{2}\left(\Delta W(n)^{\top} B \Delta W(n)-E\left[\Delta W(n)^{\top} B \Delta W(n)\right]\right) \\
& =\frac{1}{2}\left(\Delta W(n)^{\top} B \Delta W(n)-\operatorname{Tr}(B) \Delta t\right)
\end{aligned}
$$

where $B$ is the symmetric matrix with entries

$$
B_{j k}=\sum_{i=1}^{d} w_{i} \sigma_{i j} \sigma_{i k}-\sum_{i=1}^{d} w_{i} \sigma_{i j} \sum_{\ell=1}^{d} w_{\ell} \sigma_{\ell k} .
$$

We may also write the matrix $B$ as

$$
B=\sum_{i=1}^{d} w_{i} \sigma_{i} \sigma_{i}^{\top}-\bar{\sigma} \bar{\sigma}^{\top}
$$

with $\bar{\sigma}=\sum_{i} w_{i} \sigma_{i}$, as in (1).

Proposition 2 The variance parameter $\sigma_{L}^{2}$ is given by

$$
\sigma_{L}^{2}=\frac{1}{2} \operatorname{Tr}\left(B^{2}\right)=\frac{1}{2}\left(w^{\top}(\Sigma \circ \Sigma) w-2 w^{\top} \Sigma \Omega \Sigma w+\left(w^{\top} \Sigma w\right)^{2}\right)
$$

where $\circ$ denote elementwise multiplication and $\Omega$ is a diagonal matrix with $\Omega_{i i}=w_{i}, i=1, \ldots, d$.

The matrix $B$ reflects the dispersion in the weights $w$ and volatilities $\sigma_{i j}$. This can be seen in (12), which is the difference between a weighted average of products and a product of weighted averages. Representing $\sigma_{L}^{2}$ in terms of $B\left(\operatorname{Tr}\left(B^{2}\right)\right.$ is the square of the Frobenius norm of $\left.B\right)$ thus provides some insight into the parameters affecting $\sigma_{L}^{2}$. However, $B$ depends on the way we factor the covariance matrix $\Sigma$ (through the volatilities $\sigma_{i j}$ ), which is somewhat arbitrary. In contrast, the alternative representation in Proposition 2 depends only on $w$ and $\Sigma$ and thus shows that $\sigma_{L}^{2}$ is invariant with respect to the choice of factorization of $\Sigma$.

While the limiting distribution of the relative difference between the two portfolios (the second component of (7)) is normal, Theorem 1 shows that the limiting distribution of the absolute difference (the first component) is a lognormal mixture of normals. As a further illustration of the explicit nature of this limit, we record some its moments:

Proposition 3 The limiting distribution of the scaled difference $\sqrt{N}\left(\hat{V}_{N}-V(T)\right)$ has variance $\sigma_{L}^{2} T \exp \left(2 \mu_{w} T+\sigma_{w}^{2} T\right)$, kurtosis $3 \exp \left(4 \sigma_{w}^{2} T\right)$, and all odd moments equal to zero. 
Proof. The independent product $X V(T)$ has all odd moments equal to zero because $X$ has this property, and both $X$ and $V(T)$ have finite moments of all orders. The variance of the product is then $\sigma_{L}^{2} T \cdot E\left[V(T)^{2}\right]$, and $E\left[V(T)^{2}\right]=\exp \left(2 \mu_{w} T+\sigma_{w}^{2} T\right)$. For the kurtosis, we need to calculate

$$
\frac{E V(T)^{4} X^{4}}{\left(E V(T)^{2} X^{2}\right)^{2}}=\frac{E V(T)^{4} E X^{4}}{\left(E V(T)^{2} E X^{2}\right)^{2}}=3 \frac{E V(T)^{4}}{\left(E V(T)^{2}\right)^{2}},
$$

using first the independence of $V(T)$ and $X$ and then the fact that, as a normal random variable, $X$ has a kurtosis of 3 . The fourth moment of $V(T)$ is

$$
e^{4 \mu_{w} T-2 \sigma_{w}^{2} T} E\left[\exp \left(4 \bar{\sigma}^{\top} W(T)\right)\right]=e^{4 \mu_{w} T+6 \sigma_{w}^{2} T}
$$

\subsection{Example}

To illustrate the convergence in Theorem 1, we provide a numerical example. We consider a portfolio of ten assets, though these could alternatively be interpreted as subportfolios of different types of assets. The individual asset volatilities range from $2.5 \%$ to $13.75 \%$ in increments of $1.25 \%$, and all pairwise correlations of distinct assets are equal to 0.2 . The mean rate of return on the $i$ th asset is $\mu_{i}=.05+\left\|\sigma_{i}\right\| / 4$. We choose portfolio weights by maximizing the portfolio growth rate $\mu_{w}-\sigma_{w}^{2} / 2$ (which is equivalent to choosing optimal weights with log utility). This yields the weights

$$
(-5.3865,-0.7930,0.4545,0.8673,1.0025,1.0306,1.0142,0.9792,0.9373,0.8937) \text {. }
$$

We measure the portfolio's leverage as the ratio of the total long position (the sum of the positive weights) divided by the initial capital (the sum of all the weights, which is simply 1 ). This yields a leverage ratio of 7.2 . The resulting portfolio volatility is $\sigma_{w}=35.51 \%$ with a mean rate of return $\mu_{w}=18.24 \%$.

Table 1 illustrates the convergence of moments as the number of rebalancing dates $N$ increases, based on simulation estimates. The first three columns of results show estimates of the standard deviation, skewness, and kurtosis of the relative difference $X_{N}$ between the discrete and continuous portfolios; the next three columns show estimates for the difference $\hat{V}_{N}-V(T)$; and the last column shows estimates of the correlation between $X_{N}$ and $\log V(T)$. The values in the table are estimated from one million replications; the last row shows theoretical values under the limiting distributions. The standard deviations (for both the relative and absolute differences) and correlations are very close to the limiting values, even at small values of $N$. As one might expect, the higher moments are initially much farther from the values under the limiting distribution. As we will see below, this is at least partly because of the relatively high leverage ratio in this example. 


\begin{tabular}{rrrrrrrrrr} 
& \multicolumn{3}{c}{ Relative Difference $X_{N}$} & & \multicolumn{3}{c}{ Absolute Difference $\sqrt{N}\left(\hat{V}_{N}-V_{N}\right)$} & \\
\cline { 2 - 4 } \cline { 6 - 7 }$N$ & Std Dev & Skewness & Kurtosis & & Std Dev & Skewness & Kurtosis & Correl. \\
\hline 2 & 0.079 & -2.48 & 16.6 & & 0.102 & -3.41 & 28.1 & -0.003 \\
4 & 0.078 & -1.49 & 7.3 & & 0.100 & -2.56 & 18.8 & 0.002 \\
12 & 0.077 & -0.75 & 3.9 & & 0.099 & -1.57 & 10.6 & 0.000 \\
52 & 0.077 & -0.35 & 3.2 & & 0.098 & -0.77 & 6.5 & 0.001 \\
100 & 0.077 & -0.25 & 3.1 & & 0.098 & -0.57 & 5.7 & -0.002 \\
\hline$\infty$ & 0.0766 & 0 & 3 & & 0.0978 & 0 & 4.97 & 0 \\
\hline
\end{tabular}

Table 1: Numerical illustration of convergence of relative and absolute differences as the number of rebalancing dates $N$ increases. The last row shows theoretical values for the limit.
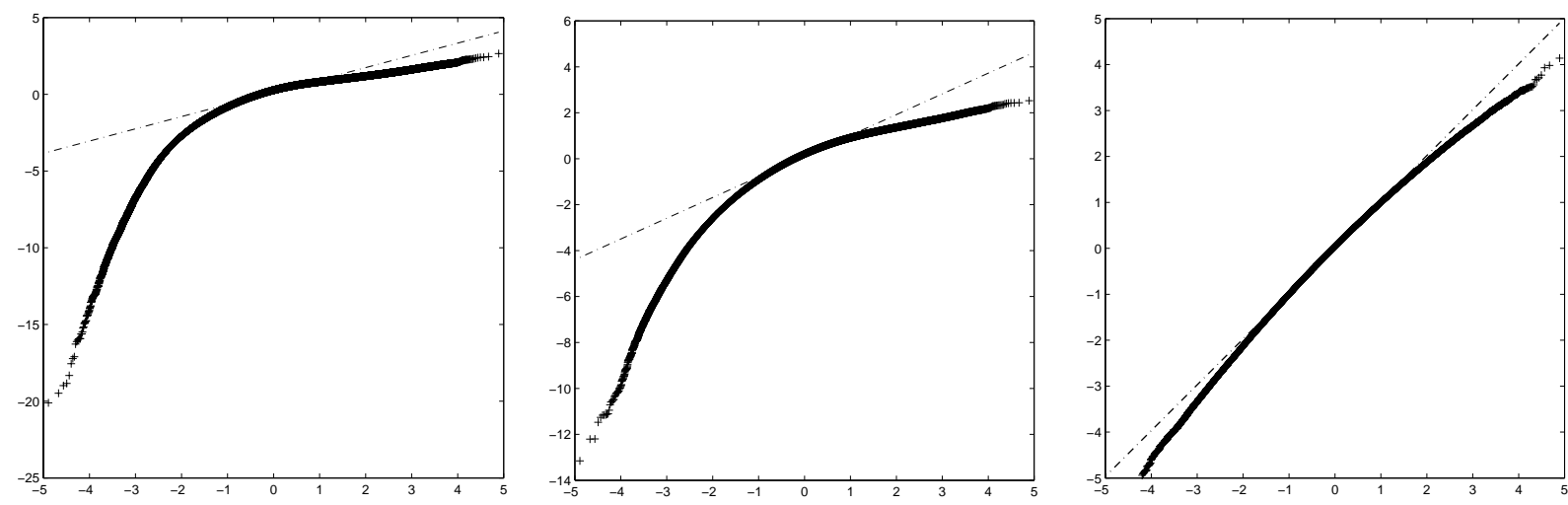

Figure 1: QQ plots of $X_{N} / \sigma_{L} T$ at $N=2$ (left), $N=4$ (middle), and $N=100$ (right), illustrating the convergence to normality.

Figure 1 illustrates the convergence to normality of the relative rebalancing error $X_{N}$, scaled by its limiting standard deviation $\sigma_{L} T$. The figure shows QQ-plots at $N=2$ (left), $N=4$ (middle), and $N=100$ (right), based on one million replications of each. (The observations would fall on a straight line if the samples conformed exactly to a normal distribution.) In this leveraged example, the distribution is initially quite negatively skewed and shows a heavy left tail, indicating a high likelihood of significant underperformance by the discretely rebalanced portfolio. The convergence to normality for large $N$ is reflected in the right panel.

Figure 2 shows scatter plots of $X_{N}$ against $\log V(T)$. At $N=2$, dependence between the two is evident, despite the low correlation reported in Table 1. The pattern in the leftmost panel of the figure reflects the quadratic relation between the increments of the rebalancing error and the asset returns. The asymptotic independence is reflected in the scatter plot in the rightmost panel, which shows results for $N=100$.

As a further illustration, we compare results with a modification of this portfolio. We adjust the model parameters to keep the overall mean and volatility $\mu_{w}$ and $\sigma_{w}$ unchanged while reducing leverage. We accomplish this by scaling each asset volatility by a factor of four, reducing each $\mu_{i}$ 

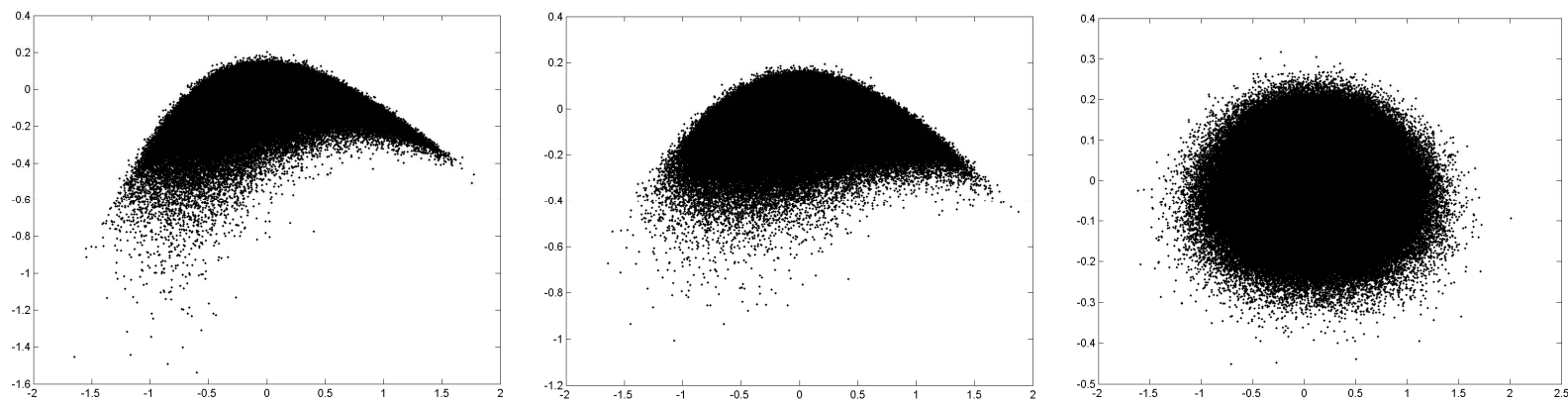

Figure 2: Scatter plots of $X_{N}$ versus $\log (V(T))$ at $N=2$ (left) and $N=100$ (right), illustrating asymptotic independence.
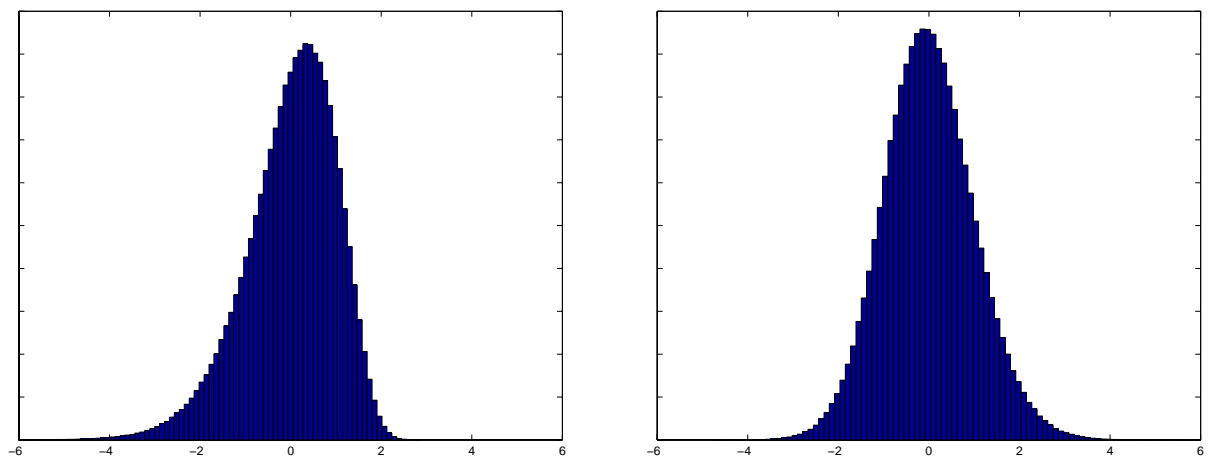

Figure 3: Histograms of $X_{N} / \sigma_{L} T$ at $N=12$ for the original (left) and reduced-leverage (right) portfolios.

by 0.0167 , and changing the weights to

$$
(-0.8036,-0.0137,0.1855,0.2424,0.2542,0.2498,0.2396,0.2274,0.2151) \text {. }
$$

(These weights maximize $\mu_{w}-c \sigma_{w}^{2} / 2$ with $c=1.029$ chosen to produce the same values of $\mu_{w}$ and $\sigma_{w}$ that we previously obtained with $c=1$.) The correlation matrix is unchanged, and the overall structure of the model is close to that of the previous example, but the resulting leverage ratio is 1.8 rather than 7.2 .

Figure 3 compares histograms of $X_{N} / \sigma_{L} T$ at $N=12$ for the original and modified models. For the portfolio with lower leverage (on the right), the distribution is closer to the normal limit and shows slightly positive skewness (estimated at 0.17 ); for the more highly leveraged portfolio (on the left), the distribution exhibits negative skewness (estimated at -0.75 ). This comparison is indicative of a more general pattern we have observed in numerical examples: the quality of the normal approximation to the relative error $X_{N}$ depends more on the degree of leverage than on the portfolio volatility or mean return, with high leverage producing negative skewness. 


\section{A Volatility Adjustment for Discrete Rebalancing}

\subsection{The Discretely Rebalanced Portfolio Distribution}

We would like to use Theorem 1 to approximate the distribution of $\hat{V}_{N}$ and then to approximate its VaR or other risk measures (as in, e.g., $[1,32]$ ). By the definition of $X_{N}$ in (9), we have

$$
\hat{V}_{N}=V(T)\left(1+\frac{X_{N}}{\sqrt{N}}\right)
$$

This represents $\hat{V}_{N}$ as nearly the product of two lognormal random variables, because $V(T)$ is lognormal, $X_{N}$ is asymptotically normal, and $1+x / \sqrt{N}=\exp (x / \sqrt{N})+O(1 / N)$. (Recall that we have joint convergence in Theorem 1.) This suggests using a lognormal approximation to $\hat{V}_{N}$. However, we cannot assume that $\hat{V}_{N}$ is positive for fixed $N$, so we cannot take its logarithm to calculate a volatility parameter. To get around this problem, we set

$$
\bar{V}_{N}=V(T) \exp \left(X_{N} / \sqrt{N}\right)=\hat{V}_{N}+O_{p}(1 / N) .
$$

Then $\bar{V}_{N}$ is positive, and its squared volatility is

$$
\frac{\operatorname{Var}\left[\log \bar{V}_{N}\right]}{T}=\frac{1}{T} \operatorname{Var}\left[\log V(T)+\frac{X_{N}}{\sqrt{N}}\right]=\sigma_{w}^{2}+\frac{\operatorname{Var}\left[X_{N}\right]}{T N}+2 \frac{\operatorname{Cov}\left[\log V(T), X_{N}\right]}{T \sqrt{N}} .
$$

For the second term on the far right of (14), Proposition 1 implies that

$$
\operatorname{Var}\left[X_{N}\right] \rightarrow \operatorname{Var}[X]=\sigma_{L}^{2} T^{2}
$$

so

$$
\frac{\operatorname{Var}\left[X_{N}\right]}{T N}=\frac{\sigma_{L}^{2} T}{N}+o(1 / N)=\sigma_{L}^{2} \Delta t+o(\Delta t) .
$$

For the last term in (14), consistent with the asymptotic independence proved in Theorem 1, we have

$$
\operatorname{Cov}\left[\log V(T), X_{N}\right] \rightarrow 0
$$

however, the rate of convergence is critical in determining whether the covariance term is negligible. It is not: the covariance is of order $O(1 / \sqrt{N})$, so the contribution of the covariance term is of the same order as the variance term.

Proposition 4 (i) We have

$$
\sqrt{N} \operatorname{Cov}\left[\log V(T), X_{N}\right] \rightarrow \gamma_{L} T^{2}
$$

where

$$
\gamma_{L}=\mu^{\top} \Omega \Sigma w-\mu_{w} \sigma_{w}^{2}+\sigma_{w}^{4}-w^{\top} \Sigma \Omega \Sigma w .
$$

(ii) $E\left[\left(\bar{V}_{N}-\hat{V}_{N}\right)^{2}\right]=O\left(N^{-2}\right)$ and

$$
N\left(\operatorname{Var}\left[\log \bar{V}_{N}\right]-\operatorname{Var}[\log V(T)]\right) \rightarrow\left(\sigma_{L}^{2}+2 \gamma_{L}\right) T^{2} .
$$


The second part of this proposition asserts that the difference between $\hat{V}_{N}$ and $\bar{V}_{N}$ is negligible (relative to our approximations) and calculates the limiting volatility of the positive sequence $\bar{V}_{N}$, which we may therefore interpret as the limiting volatility of $\hat{V}_{N}$. Armed with this limit, we arrive at a volatility adjustment that adjusts the distribution of $V(T)$ to approximate the distribution of $\hat{V}_{N}$.

Volatility Adjustment: To correct for discrete rebalancing, apply the volatility adjustment

$$
\sigma_{w} \rightarrow \sigma_{\text {adj }}=\sqrt{\sigma_{w}^{2}+\left(\sigma_{L}^{2}+2 \gamma_{L}\right) \Delta t}
$$

As one would expect, the adjustment vanishes as the rebalancing interval $\Delta t$ shrinks to zero. Moreover, the adjustment can result in either a larger or smaller volatility because the sign of $\gamma_{L}$ and its magnitude relative to $\sigma_{L}^{2}$ are indeterminate.

In a model with transaction costs, Leland [29] characterizes the price of an option using the Black-Scholes formula with an adjusted volatility, the adjustment capturing the effect of discrete hedging. The adjustment in (17) is reminiscent of Leland's adjustment (as pointed out by Jan Obloj), though there is no evident connection between the terms in the two expressions. Haug [21] reviews volatility adjustements to correct for discrete dividends in option pricing. Broadie et al. [9], Hörfelt [22], Howison and Steinberg [23], and Lai, Yao, and AitSahalia [28] derive continuity corrections for discretely monitored path-dependent options by adjusting a barrier or boundary, rather than a volatility. Dufresne [16] considers adjustments to lognormal approximations for sums of lognormal approximations.

\subsection{Examples}

To evaluate the accuracy of the volatility adjustment (17), we test its performance through simulation on several examples:

Model 1: We refer to the first example introduced in Section 3.2 as Model 1a and the modification introduced before Figure 3 (with lower leverage) as Model 1b.

Model 2: This model has five assets; as in Model 1, all off-diagonal correlations are equal to 0.2 , and each asset return is of the form $\mu_{i}=0.05+\left\|\sigma_{i}\right\| / 2$. We test various levels of volatility and leverage.

2a: Asset volatilities are $10 \%$; portfolio weights are $(-3,1,1,1,1)$.

2b: Asset volatilities are 5\%; portfolio weights are $(-4.5,-4.5,4,3,3)$.

2c: Asset volatilities are 20\%; portfolio weights are $(-1.5,1,0.5,0.5,0.5)$. 
2d: Asset volatilities are 2.5\%; portfolio weights are $(-4.5,-4.5,4,3,3)$.

2e: Asset volatilities are 50\%; portfolio weights are $(0.2,0.2,0.2,0.2,0.2)$.

Model 3: For this model, we change the correlation matrix to

$$
\left(\begin{array}{ccccc}
1 & 0.9 & 0 & 0 & 0 \\
0.9 & 1 & 0 & 0 & 0 \\
0 & 0 & 1 & 0 & 0 \\
0 & 0 & 0 & 1 & 0.9 \\
0 & 0 & 0 & 0.9 & 1
\end{array}\right)
$$

The drift vector has the same form as in Model 2, and the portfolio weights are $(-4.5,4.5$, $1,-4.5,4.5)$. In Model 3a, all asset volatilities are 10\%, and in Model 3b they are all 5\%.

Model 4: This model has ten uncorrelated assets, $\Sigma=I, \mu_{i}=10.5 \%$, and $w_{i}=10 \%$, $i=1, \ldots, 10$, with a portfolio volatility of $31.6 \%$.

Table 2 shows results for these portfolios, based on four million simulation trials. The table shows the leverage ratio, volatility $\sigma_{w}$ and rate of return $\mu_{w}$ for each portfolio; it also shows the ratio $2 \gamma_{L} / \sigma_{L}^{2}$ to indicate the relative magnitude of the two parts of the adjustment across different examples. The last three columns show the error reduction achieved by the adjusted volatility at different rebalancing frequencies, $N=2, N=4$, and $N=12$. Merely noting that the adjusted volatility is close to the estimated volatility of the discrete portfolio would be uninformative, because even $\sigma_{w}$ will be close to the discrete volatility as $N$ increases; of greater significance is measuring how much of the error in $\sigma_{w}$ is corrected by the volatility adjustment. We measure this error reduction as

$$
1-\frac{\left|\sigma_{\mathrm{adj}}-\hat{\sigma}_{N}\right|}{\left|\sigma_{w}-\hat{\sigma}_{N}\right|}
$$

where $\sigma_{\text {adj }}$ is the adjusted volatility in (17), and $\hat{\sigma}_{N}$ is the estimated "volatility" of $\hat{V}_{N}$. Thus, a value close to $100 \%$ indicates that the volatility adjustment captures nearly all of the difference in volatility between the discretely and continuously rebalanced portfolios. We calculate $\hat{\sigma}_{N}$ by taking the standard deviation of $\log \hat{V}_{N}$ across paths. It is possible for $\hat{V}_{N}$ to take negative values (though this is rare in the examples we have tested), so we simply discard negative values before taking logs. This affects the calculations at $N=2$ for Models 1a, 2a, and 2b.

The results in the table confirm a very sizeable correction from the volatility adjustment, with the adjustment often capturing nearly all the difference between the discrete and continuous volatilities. There is no a priori reason to expect the error reduction to increase with $N$ : both the numerator and denominator in our error ratio vanish as $N$ increases. Nevertheless, it is noteworthy that the values in the table show a fairly consistent pattern. The portfolios with higher leverage 


\begin{tabular}{cccccrrr} 
Model & Leverage & $\sigma_{w}$ & $\mu_{w}$ & $2 \gamma_{L} / \sigma_{L}^{2}$ & $N=2$ & $N=4$ & $N=12$ \\
\hline 1a & 7.2 & 0.355 & $18.24 \%$ & 0 & $64 \%$ & $81 \%$ & $90 \%$ \\
1b & 1.8 & 0.355 & $18.24 \%$ & -.1 & $98 \%$ & $94 \%$ & $64 \%$ \\
2a & 4 & 0.326 & $10 \%$ & 3.0 & $70 \%$ & $85 \%$ & $93 \%$ \\
2b & 10 & 0.387 & $7.50 \%$ & 3.4 & $63 \%$ & $82 \%$ & $91 \%$ \\
2c & 2.5 & 0.369 & $15 \%$ & 3.9 & $61 \%$ & $81 \%$ & $93 \%$ \\
2d & 10 & 0.193 & $6.25 \%$ & 4.0 & $91 \%$ & $94 \%$ & $86 \%$ \\
2e & 1 & 0.300 & $30 \%$ & 0 & $91 \%$ & $86 \%$ & $66 \%$ \\
3a & 10 & 0.302 & $10 \%$ & 3.3 & $77 \%$ & $90 \%$ & $98 \%$ \\
3b & 10 & 0.151 & $7.50 \%$ & 3.3 & $95 \%$ & $97 \%$ & $73 \%$ \\
4 & 1 & 0.316 & $10.50 \%$ & 0 & $99 \%$ & $99 \%$ & $99 \%$ \\
\hline
\end{tabular}

Table 2: Error reduction using the adjustment across test models. The table shows the leverage ratio, volatility $\sigma_{w}$, rate of return $\mu_{w}$, and ratio $2 \gamma_{L} / \sigma_{L}^{2}$ for each portfolio. The last three columns show the error reduction achieved by the adjusted volatility at different rebalancing frequencies.

and volatility show a marked improvement in the error reduction as $N$ increases; for the portfolios with low leverage or low volatility, the adjusted volatility is nearly exact even at $N=2$.

Figure 4 illustrates the volatility adjustment in approximating the density and distribution of Model 4 with $N=4$. The left panel shows densities of the logarithms of the portfolio values on a log scale; the density of $\log V(T)$ is normal, so the logarithm of the density yields a parabola, and this is the lowest curve on the left. The uppermost curve in the left panel shows the estimated density of $\log \hat{V}_{N}$, based on four million replications; the density values are estimated as the proportion of values falling in each of 100 equally spaced intervals. The circles show the result of applying the volatility adjustment to the density of $\log V(T)$; these coincide almost perfectly with the density values estimated under discrete rebalancing. The right panel makes an analogous comparison between the cumulative distributions of the portfolio values, the lower curve corresponding to $V(T)$, the upper curve to $\hat{V}_{N}$, and the circles to the effect of applying the volatility adjustment to $V(T)$ in approximating $\hat{V}_{N}$. In both panels, the values marked by circles are calculated by applying the volatility adjustment to the exact lognormal distribution of $V(T)$, without need for simulation.

The performance of the volatility adjustment in Figure 4 is excellent; however, we will see that this adjustment is not always sufficient to produce a good approximation. In the next section, we derive a (conditional) mean correction, with particular focus on the extreme tails. As a byproduct, this analysis produces a simple characterization of why and when the volatility adjustment suffices.

\section{Extreme Tails: Conditional Mean}

The lognormal approximation to $\hat{V}_{N}$, based on the central limit theorem in Theorem 1 should be accurate over the range of values where the normal limit $X$ provides a good approximation to 

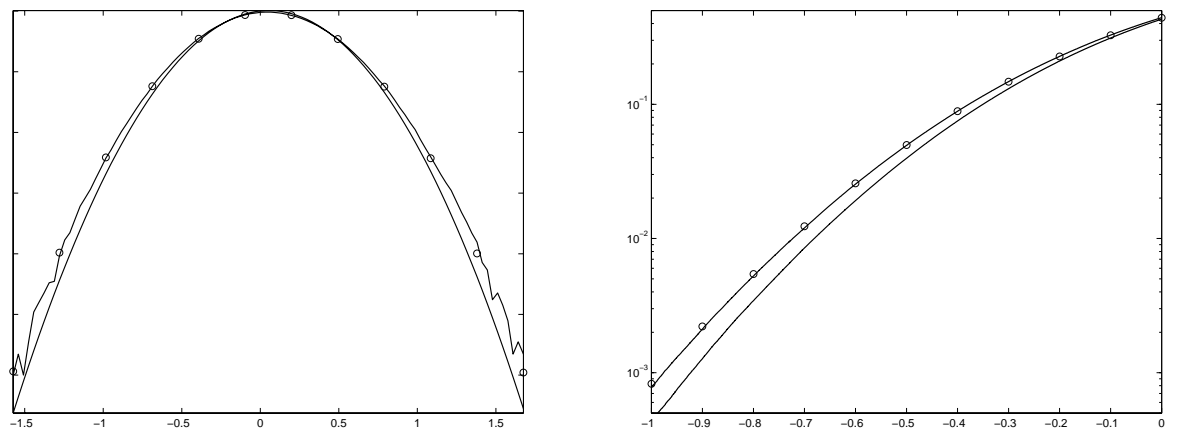

Figure 4: Density (left) and distribution (right) for Model 4. In both panels, the lower curve corresponds to the continuously rebalanced portfolio, the upper curve corresponds to the discretely rebalanced portfolio with $N=4$, and the circles are the result of applying the volatility adjustment to the continuously rebalanced distribution.

$X_{N}$. As typically happens with applications of a central limit theorem, the normal approximation loses accuracy in the extreme tails. Because the motivation for our investigation includes a $99.9 \%$ $\mathrm{VaR}$, the extreme tails are relevant. We therefore supplement the central limit theorem with an approximation specifically focused on the tails.

\subsection{Conditioning on a Large Loss}

Our approach will be to condition on an outcome of $V(T)$ in the tail of its distribution. We show that, for large $N$, the resulting conditional distribution of $\hat{V}_{N}$ is concentrated at a point: in effect, only one outcome of $\hat{V}_{N}$ is consistent with a sufficiently extreme value of $V(T)$. This limit provides a correction in approximating the distribution of $\hat{V}_{N}$ with that of $V(T)$ in the tails. It also provides an approximation to the VaR of the discretely rebalanced portfolio using that of the continuously rebalanced portfolio.

As $V(T)$ and $\bar{\sigma}^{\top} W(T)$ are in one-to-one correspondence, conditioning on a value of one is equivalent to conditioning on a value of the other. We will impose a condition of the form

$$
\bar{\sigma}^{\top} W(T)=x_{N} \equiv x \sqrt{N}, \text { for some } x \neq 0,
$$

which is equivalent to

$$
V(T)=y_{N}, \text { with } x_{N}=\log y_{N}-\mu_{w} T+\sigma_{w}^{2} T / 2 .
$$

Our choice $x_{N}=x \sqrt{N}$ is a large deviations scaling in the following sense: If we set $Z_{N, n}=$ $\Delta W(n) / \sqrt{\Delta t}$, then $\bar{\sigma}^{\top} Z_{N, n}, n=1, \ldots, N$, are independent, zero-mean normal random variables, and

$$
\frac{1}{N} \sum_{n=1}^{N} \bar{\sigma}^{\top} Z_{N, n} \Rightarrow 0
$$




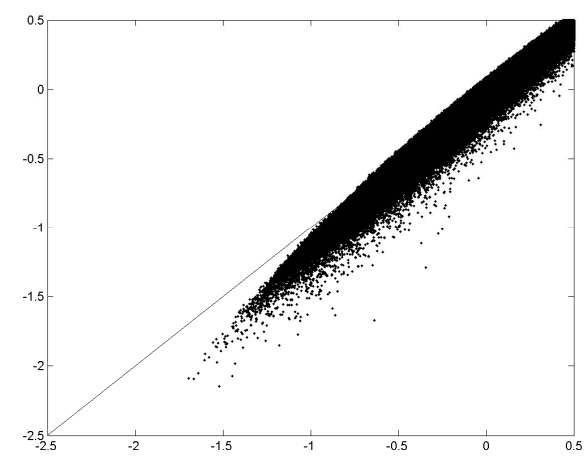

Figure 5: Scatter plot of points $\left(V(T), \hat{V}_{N}\right)$ for Model 1a.

but conditioning on $\bar{\sigma}^{\top} W(T)=x \sqrt{N}$ is equivalent to conditioning on

$$
\frac{1}{N} \sum_{n=1}^{N} \bar{\sigma}^{\top} Z_{N, n}=x / \sqrt{T} .
$$

Thus, we are conditioning a sample mean on a value away from its limit.

Theorem 2 With $x_{N}=x \sqrt{N}$ and $y_{N}=\exp \left(x_{N}+\mu_{w} T-\sigma_{w}^{2} T / 2\right)$,

$$
\left(\frac{\hat{V}_{N}}{V(T)} \mid V(T)=y_{N}\right) \Rightarrow \exp \left(\frac{1}{2} \beta_{L} x^{2}\right)
$$

as $N \rightarrow \infty$, where

$$
\beta_{L}=\frac{\bar{\sigma}^{\top} B \bar{\sigma}}{\sigma_{w}^{4}}=\frac{1}{\sigma_{w}^{4}}\left(w^{\top} \Sigma \Omega \Sigma w-\sigma_{w}^{4}\right)
$$

with $\Omega$ and $\bar{\sigma}$ as before.

The phenomenon reflected in Theorem 2 is illustrated in Figure 5. The figure shows a scatter plot of simulated pairs $\left(V(T), \hat{V}_{N}\right)$ for Model 1a, plotted on a $\log$ scale, with $T=1$ and $N=2$. The figure includes a diagonal line for comparison. Near the center of the distribution, we see substantial variability in $\hat{V}_{N}$ at each value of $V(T)$, and this variability is large relative to the differences in conditional means (i.e., the difference between the conditional mean and the diagonal line). But in the lower tail, the conditional distribution of $\hat{V}_{N}$ becomes more tightly concentrated, and the deviation from the diagonal becomes more appreciable, particularly in comparison to the conditional variability. The limit in (18) measures this deviation. The theorem applies for large $N$, but the effect is evident even at $N=2$ (a point we return to in Theorem 3 ).

The limit in Theorem 2 yields the approximation

$$
\left(\hat{V}_{N} \mid V(T)=y\right) \approx y \exp \left(\frac{1}{2} \beta_{L} x^{2} / N\right)
$$


with $x=\log y-\mu_{w} T+\frac{1}{2} \sigma_{w}^{2} T$. If we define

$$
H(y)=y \exp \left(\frac{1}{2} \beta_{L}\left(\log y-\mu_{w} T+\frac{1}{2} \sigma_{w}^{2} T\right)^{2} / N\right),
$$

then we have the approximation

$$
P\left(\hat{V}_{N} \leq y\right) \approx P\left(V(T) \leq H^{-1}(y)\right)
$$

provided $H$ is monotone near $y$. Similarly, we can approximate the VaR for the discretely rebalanced portfolio $\widehat{V a R}_{\alpha}$ (at confidence $1-\alpha$ ) in terms of that for the continuously rebalanced portfolio, $\operatorname{VaR}_{\alpha}$, using

$$
\widehat{V a R}_{\alpha} \approx H\left(\operatorname{VaR} R_{\alpha}\right)
$$

if $H$ is monotone near the VaR. At each $y>0, H^{\prime}(y)$ is positive for all sufficiently large $N$.

We can arrive at nearly the same approximation through an alternative route. Recall that we derived the central limit theorem for $\left(\hat{V}_{N}-V(T)\right) / V(T)$ by first showing that this ratio is close to the sum of the $\epsilon_{n}$. This approach suggests that we approximate the conditional expectation of the ratio (given $V(T)=y$ ) using the conditional expectation of the $\epsilon_{n}$. We can evaluate this conditional expectation exactly, without requiring that $N$ (or $y$ ) be large:

Theorem 3 For any $y>0$,

$$
E\left[\sum_{n=1}^{N} \epsilon_{n} \mid V(T)=y\right]=\frac{1}{2} \beta_{L}\left(\frac{x^{2}-\sigma_{w}^{2} T}{N}\right),
$$

where $x=\log y-\mu_{w} T+\frac{1}{2} \sigma_{w}^{2} T$.

Observe that if we let $y=y_{N}$ depend on $N$ as in Theorem 2, then we should replace $x$ with $x \sqrt{N}$, and (21) approaches $\beta_{L} x^{2} / 2$ as $N \rightarrow \infty$. This yields the approximation

$$
\left(\hat{V}_{N} \mid V(T)=y\right) \approx y\left(1+\frac{1}{2} \beta_{L} \frac{x^{2}}{N}\right)
$$

which is consistent with what we derived previously, for large $N$.

\subsection{Approximations and Examples}

Let $F\left(\cdot ; \mu, \sigma^{2}\right)$ denote a lognormal distribution with parameters $\mu$ and $\sigma^{2}$, meaning that $F\left(y ; \mu, \sigma^{2}\right)=$ $P\left(\exp \left(\mu-\sigma^{2} / 2+\sigma Z\right) \leq y\right)$, with $Z \sim N(0,1)$. Then the distribution of $V(T)$ is $F_{0}(\cdot) \equiv$ $F\left(\cdot ; \mu_{w} T, \sigma_{w}^{2} T\right)$, and this provides a crude approximation to the distribution of $\hat{V}_{N}$.

Theorem 2 provides the approximation

$$
P\left(\hat{V}_{N} \leq y\right) \approx F_{H}(y) \equiv F\left(H^{-1}(y) ; \mu_{w} T, \sigma_{w}^{2} T\right)
$$


The volatility adjustment (17) yields the approximation

$$
P\left(\hat{V}_{N} \leq y\right) \approx F_{\text {adj }}(y) \equiv F\left(y ; \mu_{w} T, \sigma_{\text {adj }}^{2} T\right),
$$

which is lognormal, but with the portfolio volatility $\sigma_{w}$ replaced by the adjusted volatility $\sigma_{\text {adj }}$.

The approximation in (22) uses an adjustment to the (conditional) mean of $\hat{V}_{N}$ (given $V(T)$ ), whereas the approximation in (23) uses an adjustment to the volatility. It is therefore natural to consider a combined approximation that uses both adjustments,

$$
P\left(\hat{V}_{N} \leq y\right) \approx F_{H, \text { adj }}(y) \equiv F\left(H^{-1}(y) ; \mu_{w} T, \sigma_{\text {adj }}^{2} T\right) .
$$

This approximation is not lognormal because of the distortion introduced by $H$.

Figure 6 illustrates these approximations for some of the test portfolios used in earlier sections. The figures show estimates, based on four million replications each, of the left tail of the portfolio value distributions, with both coordinates on logarithmic scales. In each case, the leftmost (and uppermost) solid curve shows the estimated cumulative distribution for the discretely rebalanced portfolio $\hat{V}_{N}$; the rightmost (and lowest) solid curve is $F_{0}$, the distribution of the continuously rebalanced portfolio. The dashed line shows estimated values of $F_{H}$, the adjusted distribution in (20). The correction calculated from Theorem 2 shows a substantial improvement over the crude approximation $F_{0}$ across portfolios with very different characteristics. The points marked by circles show estimated values of $F_{\text {adj }}$; in these examples, the volatility adjustment, by itself, has limited impact in the tail of the distribution. The points marked by plus sign are estimated values of $F_{H, \text { adj }}$, which combines the mean correction from Theorem 2 with the volatility adjustment from (17). This combination gives consistently excellent results, particularly at $N=12$, but even at $N=4$. This approximation is especially accurate in the important region near probabilities of the order of $10^{-3}-10^{-2}$ needed to estimate VaR at $99.9 \%$ or $99 \%$ confidence.

We have displayed values of $F_{\text {adj }}$ and $F_{H \text {,adj }}$ at just a few points (marked with circles and plus signs) in the interest of clarity; these values are just as easy to calculate as the others using the expressions in (22) and (24), so plotting a curve of values is easy. The quality of the approximations is consistent at intermediate points with the values shown in the figures. The specific points displayed were calculated as follows: we first calculated quantiles of $F_{0}$ at probabilities $10^{-5}, 10^{-4}$, $10^{-3}, 10^{-2}$, and $10^{-1}$ and then evaluated $F_{\text {adj }}$ and $F_{H, \text { adj }}$ at those points.

By itself, $F_{\text {adj }}$ (indicated with circles) is not nearly as effective in Figure 6 as it was for Model 4 in Figure 4. The results of this section explain this contrast: Model 4 has $\beta_{L}=0$. Thus, for Model $4, F_{H}=F_{0}$; the conditional mean adjustment has no effect and the volatility adjustment becomes more important. For the examples in Figure 6, the values of $\beta_{L}$ are -0.84 (Model 1a), -1.1 (Model 2a), -1.0 (Model 2b), and -.99 (Model 3a), so the mean adjustment is comparatively more significant in these examples. 

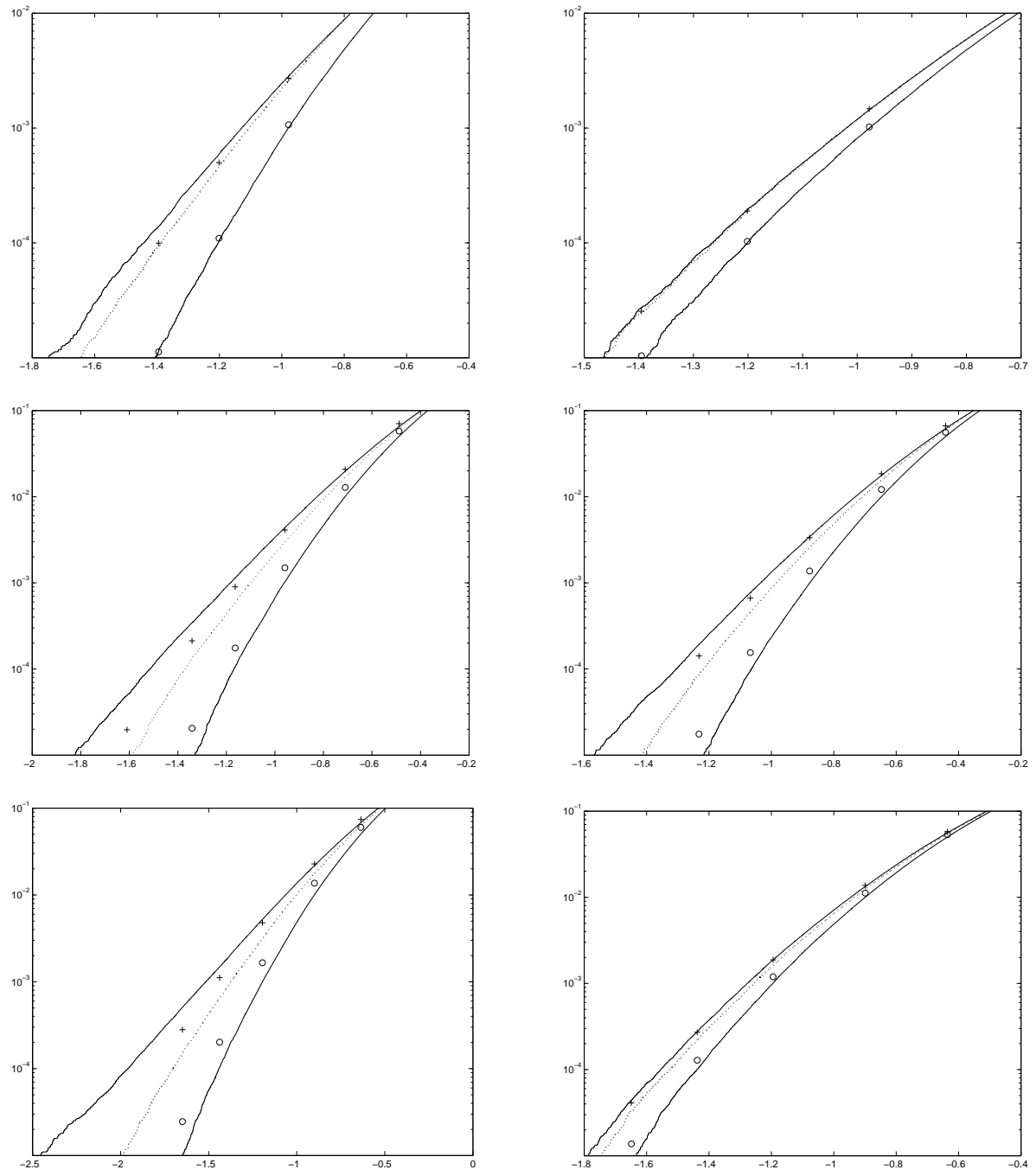

Figure 6: In each panel, the upper solid line plots the distribution of $\log \hat{V}_{N}$, and the lower solid line plots the distribution of $\log V(T)$. The dotted line is the approximation $F_{H}$, the circles show approximation $F_{\text {adj }}$, and the plus signs show approximation $F_{H \text {,adj }}$. From left to right and top to bottom, the models are 1a with $N=4$, 1a with $N=12$, 2a with $N=4$, 3a with $N=4$, 2b with $N=4$, and $2 \mathrm{~b}$ with $N=4$. 


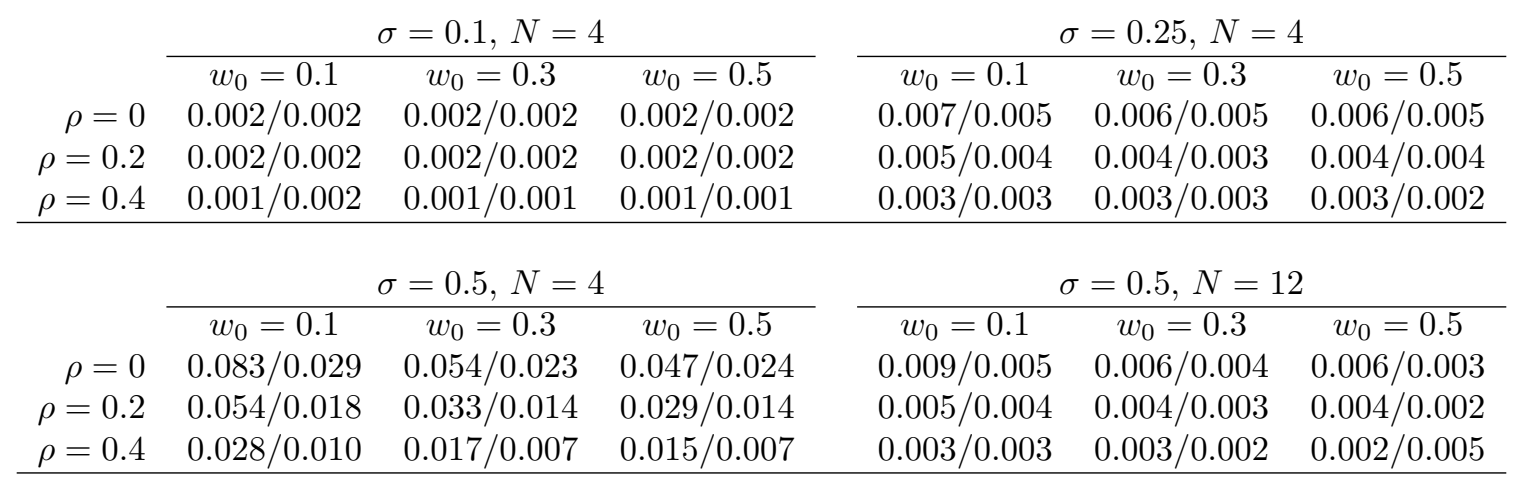

Table 3: Comparison of estimated tail probabilities. In each entry of the table, the first number gives the estimated tail probability from simulation of the discretely rebalanced portfolio, and the second number gives the approximation using $F_{H, \text { adj }}$.

Table 3 compares results under a systematic variation of model parameters. We consider a portfolio of five assets, each with volatility $\sigma$ and expected return $\mu=0.05+\sigma / 2$. Correlations between distinct assets are all equal to $\rho$. The portfolio has a leverage of two, with weights of -1 , $w_{0}, w_{0}, w_{0}$, and $2-3 w_{0}$. We compare results for $\sigma=0.1,0.25$, and $0.5 ; \rho=0,0.2$, and 0.4 ; and $w_{0}=0.1,0.3$, and 0.5 , corresponding to Herfindhal index values of $0.73,0.37$, and 0.25 , respectively, for the positive weights. There are many ways one could compare distributions across parameter values. We choose the $99.9 \%$ VaR from the continuously rebalanced distribution, estimate the tail probability for the discretely rebalanced portfolio at that loss level using simulation, and compare it with the approximation using $F_{H, \text { adj }}$.

In each entry of the table, the first number reported is the value from simulation, and the second value is the approximation. Correlation and the uniformity of the weights seem to have little impact on the accuracy of the approximation, which is generally quite good. The approximation is less accurate at high volatility with $N=4$, where it underestimates the loss probability, though the magnitude is correct. The approximation at high volatility improves at $N=12$.

\section{Subportfolios and Multiple Rebalancing Frequencies}

In this section, we examine the rebalancing error when some assets are rebalanced more frequently than others. To keep things simple, we focus on the case of two subportfolios. The analysis of this case shows how to handle the general case, so long as the rebalancing intervals for all subportfolios are integer multiples of some base interval.

Let $U_{i}(t), i=1,2$, denote the values of the subportfolios at time $t$, and let $\pi_{1}$ and $\pi_{2}=1-\pi_{1}$ denote their target weights in the full portfolio. We will use the same symbols to denote the sets of indices of the assets in each subportfolio, writing, e.g., $i \in \pi_{1}$ or $i \in \pi_{2}$ to indicate that 
the $i$ th asset is in the first or second subportfolio. We write $p_{i}$ for the weight of asset $i$ in the subportfolio that contains it, so $w_{i}=\pi_{1} p_{i}$ or $w_{i}=\pi_{2} p_{i}$, depending on whether asset $i$ is in the first or second subportfolio. We assume no asset is contained in both subportfolios; this is without loss of generality, as we could define two copies of the same asset as different assets.

Suppose, initially, that $U_{1}$ is rebalanced twice as often as $U_{2}$; its gross return over $[0, \Delta t]$ is

$$
\frac{U_{1}(\Delta t)}{U_{1}(0)}=\left(\sum_{i \in \pi_{1}} p_{i} \frac{S_{i}(\Delta t / 2)}{S_{i}(0)}\right)\left(\sum_{i \in \pi_{1}} p_{i} \frac{S_{i}(\Delta t)}{S_{i}(\Delta t / 2)}\right) .
$$

The gross return on the second subportfolio over the same period is

$$
\frac{U_{2}(\Delta t)}{U_{2}(0)}=\sum_{i \in \pi_{2}} p_{i} \frac{S_{i}(\Delta t)}{S_{i}(0)}
$$

and the gross return on the full portfolio is

$$
\pi_{1} \frac{U_{1}(\Delta t)}{U_{1}(0)}+\pi_{2} \frac{U_{2}(\Delta t)}{U_{2}(0)}
$$

The value $V(\Delta t)$ of the continuously rebalanced portfolio is unchanged from previous sections.

If we carry out a Taylor expansion of

$$
\pi_{1} \frac{U_{1}(\Delta t)}{U_{1}(0)}+\pi_{2} \frac{U_{2}(\Delta t)}{U_{2}(0)}-V(\Delta t)
$$

to terms of order $\Delta t$ (taking the magnitude of a Brownian increments to be the square root of the magnitude of the corresponding time increment), we get a quadratic function of the Brownian increments over $[0, \Delta t / 2]$ and $[\Delta t / 2, \Delta t]$, centered by its mean; this generalizes the error terms $\epsilon_{n}$ used in previous sections. For this section only, let $\Delta W_{1}$ and $\Delta W_{2}$ denote the Brownian increments over these subintervals; $\Delta W=\Delta W_{1}+\Delta W_{2}$. Then Taylor expansion of $U_{1}$ yields

$$
\frac{U_{1}(\Delta t / 2)}{U_{1}(0)} \approx\left(1+\sum_{i \in \pi_{1}} p_{i}\left(\mu_{i}-\frac{1}{2}\left\|\sigma_{i}\right\|^{2}\right) \frac{\Delta t}{2}+\sum_{i \in \pi_{1}} p_{i} \sigma_{i}^{\top} \Delta W_{1}+\frac{1}{2} \sum_{i \in \pi_{1}} p_{i}\left(\sigma_{i}^{\top} \Delta W_{1}\right)^{2}\right)
$$

and the same expression for $U_{1}(\Delta t) / U_{1}(\Delta t / 2)$ but with $\Delta W_{1}$ replaced by $\Delta W_{2}$. Similarly, we get $\frac{U_{2}(\Delta t)}{U_{2}(0)} \approx\left(1+\sum_{i \in \pi_{2}} p_{i}\left(\mu_{i}-\frac{1}{2}\left\|\sigma_{i}\right\|^{2}\right) \Delta t+\sum_{i \in \pi_{2}} p_{i} \sigma_{i}^{\top}\left(\Delta W_{1}+\Delta W_{2}\right)+\frac{1}{2} \sum_{i \in \pi_{2}} p_{i}\left(\sigma_{i}^{\top}\left(\Delta W_{1}+\Delta W_{2}\right)\right)^{2}\right)$,

and

$$
V(\Delta t) \approx 1+\left(\mu_{w}-\frac{1}{2} \sigma_{w}^{2}\right) \Delta t+\sum_{i=1}^{d} w_{i} \sigma_{i}^{\top}\left(\Delta W_{1}+\Delta W_{2}\right)+\frac{1}{2}\left(\sum_{i=1}^{d} w_{i} \sigma_{i}^{\top}\left(\Delta W_{1}+\Delta W_{2}\right)\right)^{2} .
$$

Combining these expressions and keeping only terms of order $\Delta t$ or larger yields the difference between a quadratic form in $\left(\Delta W_{1}, \Delta W_{2}\right)$ and $\theta \Delta t$, with $\theta$ as in (4). 
The limits and approximations in previous sections all result from approximating the relative difference $\left(\hat{V}_{N}-V(T)\right) / V(T)$ with the sum of the $\epsilon_{n}$. Here, we will not extend the convergence arguments given previously, but instead show how to redefine $\epsilon_{n}$ to capture different rebalancing frequencies. This provides a mechanism for extending the approximations by calculating the parameters used previously for the new increments $\epsilon_{n}$. We will illustrate this idea by evaluating their variance to generalize $\sigma_{L}^{2}$. The main difference between this setting and the previous one is that before we had only quadratics of the form $\Delta W^{\top} B \Delta W$, and now we pick up cross terms of the form $\Delta W_{1}^{\top} C \Delta W_{2}$ that arise as products of the linear (in $\Delta W_{i}$ ) terms in the expansion of $U_{1}$. These terms arise precisely because of the doubly frequent rebalancing of this subportfolio.

We use the following notation:

$$
\bar{\sigma}_{j}=\sum_{i \in \pi_{j}} p_{i} \sigma_{i}, \quad j=1,2
$$

and

$$
A_{j}=\sum_{i \in \pi_{j}} p_{i} \sigma_{i} \sigma_{i}^{\top}, \quad j=1,2 .
$$

Observe that $\bar{\sigma}=\pi_{1} \bar{\sigma}_{1}+\pi_{2} \bar{\sigma}_{2}$ and $B=\pi_{1} A_{1}+\pi_{2} A_{2}-\bar{\sigma} \bar{\sigma}^{\top}$.

Substituting (26)-(28) in (25), keeping only terms of order $O(\Delta t)$ or larger, and simplifying, we get

$\pi_{1}\left(\bar{\sigma}_{1}^{\top} \Delta W_{1}\right)\left(\bar{\sigma}_{1}^{\top} \Delta W_{2}\right)+\frac{\pi_{1}}{2} \sum_{i \in \pi_{1}} p_{i}\left(\left(\sigma_{i}^{\top} \Delta W_{1}\right)^{2}+\left(\sigma_{i}^{\top} \Delta W_{2}\right)^{2}\right)+\frac{\pi_{2}}{2} \sum_{i \in \pi_{2}} p_{i}\left(\left(\sigma_{i}^{\top} \Delta W\right)^{2}-\frac{1}{2}\left(\bar{\sigma}^{\top} \Delta W\right)^{2}-\theta \Delta t\right.$.

This, in turn, can be rewritten as

$$
\Delta W_{1}^{\top} C \Delta W_{2}+\frac{1}{2} \Delta W_{1}^{\top} B \Delta W_{1}+\frac{1}{2} \Delta W_{2}^{\top} B \Delta W_{2}-\theta \Delta t
$$

with

$$
C=\pi_{1} \bar{\sigma}_{1} \bar{\sigma}_{1}^{\top}+\pi_{2} A_{2}-\bar{\sigma} \bar{\sigma}^{\top}=B+\pi_{1}\left(\bar{\sigma}_{1} \bar{\sigma}_{1}^{\top}-A_{1}\right)
$$

The expectation of $(29)$ is

$$
0+\frac{1}{2} \operatorname{Tr}(B) \frac{\Delta t}{2}+\frac{1}{2} \operatorname{Tr}(B) \frac{\Delta t}{2}-\theta \Delta t=0
$$

By following the same steps, we can generalize this derivation to the case where the first subportfolio is rebalanced $K$ times as often as the rest of the portfolio. For this case, we divide $\Delta t$ into $K$ equally spaced subintervals and write the increment $\Delta W$ as the sum $\Delta W_{1}+\cdots+\Delta W_{K}$; (29) becomes

$$
\epsilon_{1}=\frac{1}{2} \sum_{k=1}^{K} \Delta W_{k}^{\top} B \Delta W_{k}+\sum_{j<k} \Delta W_{j}^{\top} C \Delta W_{k}-\theta \Delta t .
$$


We have labeled this $\epsilon_{1}$ because it generalizes our previous definition (the case $K=1$ ) and arises in the same way.

We calculate the variance of (31) to generalize $\sigma_{L}^{2}$. For this we need some additional notation. Arrange the indices of the assets so that all assets in the first subportfolio are listed before those in the second subportfolio. We can then partition the covariance matrix $\Sigma$ as

$$
\Sigma=\left(\begin{array}{ll}
\Sigma^{(1)} & \Sigma^{(12)} \\
\Sigma^{(21)} & \Sigma^{(2)}
\end{array}\right)=\left(\begin{array}{c}
\Sigma^{(1 \cdot)} \\
\Sigma^{(2 \cdot)}
\end{array}\right)=\left(\Sigma^{(\cdot 1)} \Sigma^{(\cdot 2)}\right) .
$$

Write $p^{(1)}$ for the vector of weights $p_{i}, i \in \pi_{1}$, and let $\Omega^{(1)}$ denote the diagonal matrix with the elements of $p^{(1)}$ on its diagonal. Let

$$
\sigma_{\pi_{1}}^{2}=p^{(1) \top} \Sigma^{(1)} p^{(1)}=\bar{\sigma}_{1}^{\top} \bar{\sigma}_{1}
$$

and define

$$
\sigma_{L}^{2}(1)=\frac{1}{2}\left(p^{(1) \top} \Sigma^{(1)} \circ \Sigma^{(1)} p^{(1)}-2 p^{(1) \top} \Sigma^{(1)} \Omega^{(1)} \Sigma^{(1)} p^{(1)}+\sigma_{\pi_{1}}^{4}\right)
$$

and

$$
\sigma_{L}^{2}(1 \cdot)=\frac{1}{2}\left(p^{(1) \top} \Sigma^{(1 \cdot)} \circ \Sigma^{(1 \cdot)} w-p^{(1) \top} \Sigma^{(1)} \Omega^{(1)} \Sigma^{(1)} p^{(1)}-w^{\top} \Sigma^{(\cdot 1)} \Omega^{(1)} \Sigma^{(1 \cdot)} w+\left(w^{\top} \Sigma^{(\cdot 1)} p^{(1)}\right)^{2}\right) .
$$

Proposition 5 We have

$$
\operatorname{Tr}\left(C^{2}\right)=2 \sigma_{L}^{2}+2 \pi_{1}^{2} \sigma_{L}^{2}(1)-2 \pi_{1} \sigma_{L}^{2}(1 \cdot)
$$

and

$$
\begin{aligned}
\operatorname{Var}\left[\epsilon_{1}\right] & =\left(\frac{\operatorname{Tr}\left(B^{2}\right)}{2}+(K-1) \frac{\operatorname{Tr}\left(C^{2}\right)}{2}\right) \frac{\Delta t^{2}}{K} \\
& =\sigma_{L}^{2} \Delta t^{2}+\frac{K-1}{K}\left(\pi_{1}^{2} \sigma_{L}^{2}(1)-\pi_{1} \sigma_{L}^{2}(1 \cdot)\right) \Delta t^{2}
\end{aligned}
$$

Of these expressions, (33) is easier to read, but $B$ and $C$ depend on the way we factor $\Sigma$ (through the vectors $\sigma_{i}$ ) whereas (34) does not. In both cases, the first term results from squared Brownian increments, and the second term results from cross products of increments over subintervals of length $\Delta t / K$ within the same interval of length $\Delta t$. When $K=1$, we recover $\sigma_{L}^{2} \Delta t^{2}$. If the first subportfolio encompasses the full portfolio, then $\pi_{1}=1, \pi_{2}=0$, and $C=0$, as one can see from the first expression in (30), so (33) reduces to $\sigma_{L}^{2} \Delta t^{2} / K$. (We have $\sigma_{L}^{2} \Delta t^{2} / K$ rather than $\sigma_{L}^{2}(\Delta t / K)^{2}$ because $\epsilon_{1}$ is evaluated at time $\Delta t$ rather than $\Delta t / K$.) If the first subportfolio is empty, then $\pi_{1}=0, \pi_{2}=1$, and $C=B$ (see the second expression in (30)), so (33) reduces to $\sigma_{L}^{2} \Delta t^{2}$. 


\section{Concluding Remarks}

Our investigation provides simple and explicit characterizations of the impact of the rebalancing constraints in the Basel Committee's incremental risk charge for market risk. We have identified an asymptotic variance parameter $\sigma_{L}^{2}$ that quantifies the magnitude of the relative difference between discretely and continuously rebalanced portfolios. In combination with an asymptotic covariance parameter $\gamma_{L}$, this yields a volatility adjustment to approximate a discretely rebalanced portfolio with a continuously rebalanced portfolio. A conditional mean adjustment parameter $\beta_{L}$ improves the approximation, particularly in the region of the loss distribution near a $99.9 \% \mathrm{VaR}$. All of these parameters are given explicitly in terms of the volatilites, correlations, and expected returns of the underlying assets.

The analysis in this paper is limited to assets modeled as correlated geometric Brownian motions. Understanding the impact of other asset dynamics is a topic for further investigation. In preliminary tests using bootstrapped returns from historical market data, we find that the moment corrections

$\sigma_{L}^{2}$ and $\gamma_{L}$ perform quite well; as one would expect, the distribution of the normalized error exhibits heavier tails than one finds with the normal distribution.

Acknowledgments. I am grateful to John Kambhu of the Federal Reserve Bank of New York for helpful discussions concerning the incremental risk charge and for comments that motivated the analysis in Section 4.1. I thank the research staff of the New York Fed, particularly Tobias Adrian, Til Schuermann, and Zhenyu Wang for their hospitality and helpful discussions. I thank the referees for their comments.

\section{Appendix}

\section{A.1 Proof of Proposition 1}

Define

$$
R_{n}=\exp \left(\mu_{w} \Delta t-\sigma_{w}^{2} \Delta t / 2+\bar{\sigma}^{\top} \Delta W(n)\right)
$$

and

$$
\hat{R} n=\sum_{i=1}^{d} w_{i} \exp \left(\mu_{i} \Delta t-\left\|\sigma_{i}\right\|^{2} \Delta t / 2+\sigma_{i}^{\top} \Delta W(n)\right)
$$

Then

$$
\frac{\hat{V}_{N}}{V_{N}}=\prod_{n=1}^{N} \frac{\hat{R}_{n}}{R_{n}}
$$

and

$$
\frac{\hat{R}_{n}}{R_{n}}=\sum_{i=1}^{d} w_{i} \exp \left(\left(\mu_{i}-\mu_{w}\right) \Delta t-\frac{1}{2}\left\|\sigma_{i}\right\|^{2} \Delta t+\frac{1}{2} \sigma_{w}^{2} \Delta t+\left(\sigma_{i}-\bar{\sigma}\right)^{\top} \Delta W(n)\right) .
$$


Let $Z_{N, n}=\Delta W(n) / \sqrt{\Delta t}$, so that, for each $N, Z_{N, 1}, \ldots, Z_{N, N}$ are independent $N(0, I)$ random vectors. Define

$$
g_{N, n}(x)=\sum_{i=1}^{d} w_{i} \exp \left(\left(\mu_{i}-\mu_{w}\right) x^{2}-\frac{1}{2}\left\|\sigma_{i}\right\|^{2} x^{2}+\frac{1}{2} \sigma_{w}^{2} x^{2}+\left(\sigma_{i}-\bar{\sigma}\right)^{\top} Z_{N, n} x\right)-1 .
$$

Then

$$
\frac{\hat{V}_{N}}{V_{N}}=\prod_{n=1}^{N}\left[1+g_{N, n}(\sqrt{\Delta t})\right]
$$

and

$$
\sum_{n=1}^{N} \epsilon_{n}=\frac{1}{2} \sum_{n=1}^{N} g_{N, n}^{\prime \prime}(0) \Delta t
$$

We apply a Taylor approximation to $g_{N, n}$. Observe first that

$$
g_{N, n}^{\prime}(0)=\sum_{i} w_{i}\left(\sigma_{i}-\bar{\sigma}\right) Z_{N, n}=0 .
$$

Then, for some $\xi_{N, n} \in[0, \sqrt{\Delta t}]$, we have

$$
\begin{aligned}
\frac{\hat{V}_{N}}{V_{N}} & =\prod_{n=1}^{N}\left[1+\frac{1}{2} g_{N, n}^{\prime \prime}(0) \Delta t+\frac{1}{6} g_{N, n}^{\prime \prime \prime}(0) \Delta t^{3 / 2}+\frac{1}{24} g_{N, n}^{(4)}\left(\xi_{N, n}\right) \Delta t^{2}\right] \\
& =1+\frac{1}{2} \sum_{n=1}^{N} g_{N, n}^{\prime \prime}(0) \Delta t+\frac{1}{6} \sum_{n=1}^{N} g_{N, n}^{\prime \prime \prime}(0) \Delta t^{3 / 2}+\frac{1}{24} \sum_{n=1}^{N} g_{N, n}^{(4)}\left(\xi_{N, n}\right) \Delta t^{2}+r_{N}
\end{aligned}
$$

where the remainder $r_{N}$ includes all other terms in the product. In light of (38), the proof will be concluded once we show that the last three terms in (40) are indeed negligible.

In the following, when applied to a random variable the symbol $\|\cdot\|$ denotes the $L^{2}$ norm, $\|X\|=\left(E\left[X^{2}\right]\right)^{1 / 2}$. In light of (40) and (38), we have

$$
\left\|\frac{\hat{V}_{N}-V_{N}}{V_{N}}-\sum_{n=1}^{N} \epsilon_{n}\right\| \leq\left\|\frac{1}{6} \sum_{n=1}^{N} g_{N, n}^{\prime \prime \prime}(0) \Delta t^{3 / 2}\right\|+\left\|\frac{1}{24} \sum_{n=1}^{N} g_{N, n}^{(4)}\left(\xi_{N, n}\right) \Delta t^{2}\right\|+\left\|r_{N}\right\| .
$$

We discuss the terms on the right in turn, showing that each is $O(\Delta t)$.

Direct calculation shows that $g_{N, n}^{\prime \prime \prime}(0)$ is a linear combination of terms $\left(\sigma_{i}-\bar{\sigma}\right)^{\top} Z_{N, n}$ and $\left[\left(\sigma_{i}-\right.\right.$ $\left.\bar{\sigma})^{\top} Z_{N, n}\right]^{3}$, and thus has mean zero. (Indeed, for all $j=1,2, \ldots, g_{N, n}^{(j)}(0)$ is a linear combination of odd powers of normal random variables if $j$ is odd and even powers of normal random variables if $j$ is even.) Thus, the first term on the right in (41) is the norm of a sum of uncorrelated (in fact, independent), identically distributed, mean zero random variables. It follows that

$$
\left\|\frac{1}{6} \sum_{n=1}^{N} g_{N, n}^{\prime \prime \prime}(0) \Delta t^{3 / 2}\right\|^{2}=\frac{N}{36} \operatorname{Var}\left[g_{N, 1}^{\prime \prime \prime}(0)\right] \Delta t^{3}=O\left(\Delta t^{2}\right),
$$


and thus the norm is $O(\Delta t)$.

In the next term in (41), the summands do not have mean zero, but the triangle inequality yields

$$
\left\|\frac{1}{24} \sum_{n=1}^{N} g_{N, n}^{(4)}\left(\xi_{N, n}\right) \Delta t^{2}\right\| \leq \frac{N}{24}\left\|g_{N, n}^{(4)}\left(\xi_{N, n}\right)\right\| \Delta t^{2}=O\left(N \Delta t^{2}\right)=O(\Delta t) .
$$

The norm of $g_{N, n}^{(4)}\left(\xi_{N, n}\right)$ is finite because $\xi_{N, n} \in[0, \sqrt{\Delta t}]$ and $g^{(4)}$ is continuous.

It remains to show that we have $\left\|r_{N}\right\|=O(\Delta t)$ for the remainder in (41). Each term in $r_{N}$ is a product of the four types of terms in (39). We group the terms in $r_{N}$ according to the number $J$ of factors different from 1 , for $J=2,3, \ldots, N$. The cases $J=0$ and $J=1$ appear explicitly in (40). To lighten notation, we write

$$
a_{n}=g_{N, n}^{\prime \prime}(0) / 2, \quad b_{n}=g_{N, n}^{\prime \prime \prime}(0) / 6, \quad c_{n}=g_{N, n}^{(4)}\left(\xi_{N, n}\right) / 24,
$$

for $n=1, \ldots, N$. Then

$$
r_{N}=\sum_{J=2}^{N} \sum_{k=0}^{J} \sum_{\ell=0}^{J-k} \sum_{n_{1}, \ldots, n_{J}} c_{n_{1}} \cdots c_{n_{k}} b_{n_{k+1}} \cdots b_{n_{k+\ell}} a_{n_{k+\ell+1}} \cdots a_{n_{J}} \Delta t^{2 k} \Delta t^{3 \ell / 2} \Delta t^{J-k-\ell},
$$

where, in the innermost sum, each $n_{i}$ ranges from 1 to $N$ and the sum is taken over sets of distinct indices $n_{1}, \ldots, n_{J}$. In this expression for $r_{N}$, we have collected powers of $\Delta t$ according to their sources in (39): the $k$ factors $c_{n_{1}}, \ldots, c_{n_{k}}$ contribute $\Delta t^{2 k}$, and so on.

Because the sum in (42) is over sets of distinct indices, each product in the sum is a product of independent random variables drawn from up to three distributions. Thus, we have

$\left\|c_{n_{1}} \cdots c_{n_{k}} b_{n_{k+1}} \cdots b_{n_{k+\ell}} a_{n_{k+\ell+1}} \cdots a_{n_{J}}\right\|=\left\|c_{n_{1}}\right\| \cdots\left\|c_{n_{k}}\right\|\left\|b_{n_{k+1}}\right\| \cdots\left\|b_{n_{k+\ell}}\right\|\left\|a_{n_{k+\ell+1}}\right\| \cdots\left\|a_{n_{J}}\right\| \leq \rho^{J}$

for some $\rho$ not depending on $J$ or $N$; e.g., if $\Delta t<1$, we can take

$$
\rho=\max \left(\left\|a_{1}\right\|,\left\|b_{1}\right\|, \max _{0 \leq \tau \leq 1}\left\|g_{N, 1}^{(4)}(\tau)\right\|\right)<\infty .
$$

We decompose the remainder in (42) as $r_{N}^{(0)}+r_{N}^{(1)}$ by letting $r_{N}^{(0)}$ be the sum in (42) restricted to terms with $k=0$ and letting $r_{N}^{(1)}$ be the sum over terms with $k \geq 1$. We will bound the norm of each of these terms. For $r_{N}^{(0)}$, we have

$$
r_{N}^{(0)}=\sum_{J=2}^{N} \sum_{\ell=0}^{J} \sum_{n_{1}, \ldots, n_{J}} b_{n_{1}} \cdots b_{n_{\ell}} a_{n_{\ell+1}} \cdots a_{n_{J}} \Delta t^{J} \Delta t^{\ell / 2} .
$$

We claim that this is a sum of uncorrelated, mean zero terms. First observe that, for all $n, m=$ $1, \ldots, N$ with $m \neq n$,

$$
E\left[a_{n} b_{n}\right]=0 \text { and } E\left[a_{n} a_{m}\right]=E\left[b_{n} b_{m}\right]=E\left[a_{n} b_{m}\right]=0 .
$$


To verify the first equality, recall that each $b_{n}$ is a linear combination of odd powers of $\left(\sigma_{i}-\bar{\sigma}\right)^{\top} Z_{N, n}$ and each $a_{n}$ is a centered sum of even powers. The other equalities from the independence of the $Z_{N, n}, n=1, \ldots, N$. Thus, each summand in $r_{N}^{(0)}$ has mean zero, and the product of any two distinct summands also has mean zero. The squared norm of $r_{N}^{(0)}$ (its variance) then satisfies

$$
\begin{aligned}
\left\|r_{N}^{(0)}\right\|^{2} & =\sum_{J=2}^{N} \sum_{\ell=0}^{J} \sum_{n_{1}, \ldots, n_{J}}\left\|b_{n_{1}} \cdots b_{n_{\ell}} a_{n_{\ell+1}} \cdots a_{n_{J}}\right\|^{2} \Delta t^{2 J+\ell} \\
& \leq \sum_{J=2}^{N} \sum_{\ell=0}^{J}\left(\begin{array}{c}
N \\
J
\end{array}\right)\left(\begin{array}{l}
J \\
\ell
\end{array}\right) \rho^{2 J} \Delta t^{2 J+\ell} \\
& =\sum_{J=2}^{N}\left(\begin{array}{c}
N \\
J
\end{array}\right) \rho^{2 J} \Delta t^{2 J}(1+\Delta t)^{J} \\
& \sim\left(\begin{array}{c}
N \\
2
\end{array}\right) \rho^{4} \Delta t^{4}(1+\Delta t)^{2} \sim \rho^{4} T^{2} \Delta t^{2} / 2
\end{aligned}
$$

where " $\sim$ " means that the ratio of the two sides approaches 1 as $N \rightarrow \infty$. Thus, $\left\|r_{N}^{(0)}\right\|=O(\Delta t)$.

The remaining term is

$$
r_{N}^{(1)}=\sum_{J=2}^{N} \sum_{k=1}^{J} \sum_{\ell=0}^{J-k} \sum_{n_{1}, \ldots, n_{J}} c_{n_{1}} \cdots c_{n_{k}} b_{n_{k+1}} \cdots b_{n_{k+\ell}} a_{n_{k+\ell+1}} \cdots a_{n_{J}} \Delta t^{J+k+\ell / 2},
$$

The triangle inequality yields

$$
\begin{aligned}
\left\|r_{N}^{(1)}\right\| & \leq \sum_{J=2}^{N} \sum_{k=1}^{J} \sum_{\ell=0}^{J-k} \sum_{n_{1}, \ldots, n_{J}}\left\|c_{n_{1}} \cdots c_{n_{k}} b_{n_{k+1}} \cdots b_{n_{k+\ell}} a_{n_{k+\ell+1}} \cdots a_{n_{J}}\right\| \Delta t^{J+k+\ell / 2} \\
& \leq \sum_{J=2}^{N} \sum_{k=1}^{J} \sum_{\ell=0}^{J-k}\left(\begin{array}{c}
N \\
J
\end{array}\right)\left(\begin{array}{c}
J \\
k
\end{array}\right)\left(\begin{array}{c}
J-k \\
\ell
\end{array}\right) \rho^{J} \Delta t^{J+k+\ell / 2} \\
& =\sum_{J=2}^{N} \sum_{k=1}^{J}\left(\begin{array}{l}
N \\
J
\end{array}\right)\left(\begin{array}{l}
J \\
k
\end{array}\right) \rho^{J} \Delta t^{J+k}\left(1+\Delta t^{1 / 2}\right)^{J-k} \\
& =\sum_{J=2}^{N}\left(\begin{array}{c}
N \\
J
\end{array}\right) \rho^{J} \Delta t^{J}\left[\left(1+\Delta t^{1 / 2}+\Delta t\right)^{J}-\left(1+\Delta t^{1 / 2}\right)^{J}\right] \\
& =\left[1+\rho \Delta t\left(1+\Delta t^{1 / 2}+\Delta t\right)\right]^{N}-\left[1+\rho \Delta t\left(1+\Delta t^{1 / 2}\right)\right]^{N}-N \rho \Delta t^{2} \\
& \sim \rho T(\exp (\rho T)-1) \Delta t
\end{aligned}
$$

as $N \rightarrow \infty$. The last step uses the fact that $(1+x / N)^{N} \rightarrow \exp (x)$. Thus, $\left\|r_{N}^{(1)}\right\|=O(\Delta t)$, and then $\left\|r_{N}\right\| \leq\left\|r_{N}^{(0)}\right\|+\left\|r_{N}^{(1)}\right\|$ is also $O(\Delta t)$.

\section{A.2 Proof of Proposition 2}

With $Z$ a $d$-dimensional standard normal vector, we have, for any $d \times d$ matrix $A$,

$$
\operatorname{Var}\left[Z^{\top} A Z\right]=2 \operatorname{Tr}\left(A^{\top} A\right)
$$


so the first expression for $\sigma_{L}^{2}$ follows from rewriting (6) as $\sigma_{L}^{2}=\operatorname{Var}\left[Z^{\top} B Z / 2\right]$ and observing that $B$ is symmetric.

Squaring $B$ yields three terms; we evaluate $\operatorname{Tr}\left(B^{2}\right)$ as the sum of the traces of these terms:

$$
\begin{aligned}
\left.\operatorname{Tr}\left(\sum_{i=1}^{d} w_{i} \sigma_{i} \sigma_{i}^{\top}\right)^{2}\right)=\operatorname{Tr}\left(\sum_{i, j=1}^{d} w_{i} w_{j} \sigma_{i} \sigma_{i}^{\top} \sigma_{j} \sigma_{j}^{\top}\right) & =\sum_{i, j=1}^{d} w_{i} w_{j} \operatorname{Tr}\left(\sigma_{i} \Sigma_{i j} \sigma_{j}^{\top}\right) \\
& =\sum_{i, j=1}^{d} w_{i} w_{j} \operatorname{Tr}\left(\sigma_{j}^{\top} \sigma_{i} \Sigma_{i j}\right) \\
& =\sum_{i, j=1}^{d} w_{i} w_{j}\left(\Sigma_{i j}\right)^{2}=w^{\top} \Sigma \circ \Sigma w ; \\
\left.-2 \operatorname{Tr}\left(\sum_{i=1}^{d} w_{i} \sigma_{i} \sigma_{i}^{\top}\right) \bar{\sigma} \bar{\sigma}^{\top}\right) & =-2 \sum_{i=1}^{d} w_{i} \operatorname{Tr}\left(\sigma_{i} \sigma_{i}^{\top} \bar{\sigma} \bar{\sigma}^{\top}\right) \\
& =-2 \sum_{i=1}^{d} w_{i}\left(\bar{\sigma}^{\top} \sigma_{i}\right)^{2} \\
& =-2 \sum_{i=1}^{d} w_{i}\left(\sum_{\ell} w_{\ell} \sigma_{\ell}^{\top} \sigma_{i}\right)^{2} \\
& =-2 \sum_{i=1}^{d} w_{i}\left(\sum_{\ell} w_{\ell} \Sigma_{\ell i}\right)^{2}=-2 w^{\top} \Sigma \Omega \Sigma w . \\
\operatorname{Tr}\left(\bar{\sigma} \bar{\sigma}^{\top} \bar{\sigma} \bar{\sigma}^{\top}\right)= & \operatorname{Tr}\left(\bar{\sigma}^{\top} \bar{\sigma} \bar{\sigma}^{\top} \bar{\sigma}\right)=\left(\bar{\sigma}^{\top} \bar{\sigma}\right)^{2}=\sigma_{w}^{4} ;
\end{aligned}
$$

Adding (44), (46) and (47) and multiplying by $1 / 2$ yields the result.

\section{A.3 Proof of Proposition 4}

(i) From (2), we have

$$
X_{N}=\sqrt{N} \sum_{n=0}^{N-1}\left(\frac{\hat{V}_{n+1}}{V_{n+1}}-\frac{\hat{V}_{n}}{V_{n}}\right)
$$

so

$$
\operatorname{Cov}\left[\log V_{N}, X_{N}\right]=\sqrt{N} \sum_{k=1}^{N} \sum_{n=0}^{N-1} E\left[\bar{\sigma}^{\top} \Delta W(k)\left(\frac{\hat{V}_{n+1}}{V_{n+1}}-\frac{\hat{V}_{n}}{V_{n}}\right)\right] .
$$

We claim that

$$
E\left[\bar{\sigma}^{\top} \Delta W(k)\left(\frac{\hat{V}_{n+1}}{V_{n+1}}-\frac{\hat{V}_{n}}{V_{n}}\right)\right]= \begin{cases}0, & k \geq n+2 \\ \gamma_{L} \Delta t^{2}+O\left(\Delta t^{3}\right), & k=n+1 \\ O\left(\Delta t^{4}\right), & k \leq n\end{cases}
$$


The first case in (49) follows immediately from the independence of $\Delta W(k)$ and $\left(\hat{V}_{n}, V_{n}, \hat{V}_{n+1}, V_{n+1}\right)$, for $k \geq n+2$. For the second case in (49), we may write

$$
E\left[\bar{\sigma}^{\top} \Delta W(k)\left(\frac{\hat{V}_{n+1}}{V_{n+1}}-\frac{\hat{V}_{n}}{V_{n}}\right)\right]=E\left[\frac{\hat{V}_{n}}{V_{n}}\right] E\left[\bar{\sigma}^{\top} \Delta W(k) \frac{\hat{R}_{n+1}}{R_{n+1}}\right] .
$$

Now

$$
\begin{aligned}
& E\left[\bar{\sigma}^{\top} \Delta W(n+1) \frac{\hat{R}_{n+1}}{R_{n+1}}\right] \\
& =\sum_{i=1}^{d} w_{i} E\left[\bar{\sigma}^{\top} \Delta W(n+1) \exp \left(\left[\mu_{i}-\mu_{w}-\frac{1}{2}\left\|\sigma_{i}\right\|^{2}+\frac{1}{2} \sigma_{w}^{2}\right] \Delta t+\left(\sigma_{i}-\bar{\sigma}\right)^{\top} \Delta W(n+1)\right)\right] \\
& =\sum_{i=1}^{d} w_{i}\left(\bar{\sigma}^{\top} \sigma_{i}-\sigma_{w}^{2}\right) \Delta t \exp \left(\left(\mu_{i}-\mu_{w}+\sigma_{w}^{2}-\sigma_{i}^{\top} \bar{\sigma}\right) \Delta t\right) .
\end{aligned}
$$

The last equality uses $\bar{\sigma}^{\top} \bar{\sigma}=\sigma_{w}^{2}$ and the identity

$$
E\left[\bar{\sigma}^{\top} \Delta W(n+1) \exp \left(-\frac{1}{2}\left\|\left(\sigma_{i}-\bar{\sigma}\right)^{\top}\right\|^{2} \Delta t+\left(\sigma_{i}-\bar{\sigma}\right)^{\top} \Delta W(n+1)\right)\right]=\bar{\sigma}^{\top}\left(\sigma_{i}-\bar{\sigma}\right) \Delta t ;
$$

this follows from Girsanov's theorem, as the exponential in this expectation is the density for a change of measure that changes the mean of $\Delta W(n+1)$ to $\left(\sigma_{i}-\bar{\sigma}\right) \Delta t$. Expanding $(51)$, we find that the $O(\Delta t)$ term is actually zero because its coefficient is

$$
\sum_{i=1}^{d} w_{i}\left(\bar{\sigma}^{\top} \sigma_{i}-\sigma_{w}^{2}\right)=\bar{\sigma}^{\top} \bar{\sigma}-\sigma_{w}^{2}=0 .
$$

The coefficient on $\Delta t^{2}$ is

$$
\begin{aligned}
\sum_{i=1}^{d} w_{i}\left(\mu_{i}-\mu_{w}+\sigma_{w}^{2}-\sigma_{i}^{\top} \bar{\sigma}\right)\left(\sigma_{i}^{\top} \bar{\sigma}\right) & =\sum_{i=1}^{d} w_{i}\left(\mu_{i}-\mu_{w}\right) \sigma_{i}^{\top} \bar{\sigma}+\sigma_{w}^{4}-\sum_{i} w_{i}\left(\sigma_{i}^{\top} \bar{\sigma}\right)^{2} \\
& =\sum_{i=1}^{d} w_{i} \mu_{i} \sigma_{i}^{\top} \bar{\sigma}-\mu_{w} \sigma_{w}^{2}+\sigma_{w}^{4}-\sum_{i} w_{i}\left(\sigma_{i}^{\top} \bar{\sigma}\right)^{2} \\
& =\gamma_{L},
\end{aligned}
$$

and the remainder is $O\left(\Delta t^{3}\right)$.

By essentially the same argument,

$$
E\left[\frac{\hat{V}_{n}}{V_{n}}\right]=\prod_{k=1}^{n} E\left[\frac{\hat{R}_{k}}{R_{k}}\right]=\prod_{k=1}^{n}\left(1+O\left(\Delta t^{2}\right)\right)=1+O(\Delta t),
$$

so (50) is indeed $\gamma_{L} \Delta t^{2}+O\left(\Delta t^{3}\right)$. 
Using the independence of the Brownian increments, we can write the last case in (49) as

$$
E\left[\frac{\hat{V}_{k-1}}{V_{k-1}}\right] E\left[\bar{\sigma}^{\top} \Delta W(k) \frac{\hat{R}_{k}}{R_{k}}\right]\left(\prod_{m=k+1}^{n} E\left[\frac{\hat{R}_{m}}{R_{m}}\right]\right) E\left[\frac{\hat{R}_{n+1}}{R_{n+1}}-1\right] .
$$

With the calculations above, this is

$$
(1+O(\Delta t)) \cdot O\left(\Delta t^{2}\right) \cdot(1+O(\Delta t)) \cdot O\left(\Delta t^{2}\right)=O\left(\Delta t^{4}\right)
$$

Applying (49) to (48), we get

$$
N^{-1 / 2} \operatorname{Cov}\left[\log V(T), X_{N}\right]=O\left(N^{2}\right) \cdot O\left(\Delta t^{4}\right)+N \cdot\left(\gamma_{L} \Delta t^{2}+O\left(\Delta t^{3}\right)\right)=\frac{\gamma_{L} T^{2}}{N}+O\left(N^{-2}\right),
$$

which implies (15).

For part (ii) of the proposition, the mean value theorem yields

$$
\bar{V}_{N}-\hat{V}_{N}=V(T)\left(\exp \left(X_{N} / \sqrt{N}\right)-1-\frac{X_{N}}{\sqrt{N}}\right)=V(T) \cdot \frac{1}{2} \frac{\xi_{N}}{N}
$$

for some $\xi_{N}$ with $\left|\xi_{N}\right| \leq\left|X_{N}\right|$. We need to show that $E\left[V(T)^{2} \xi_{N}^{2}\right]$ is bounded, and this will follow if we show that $E\left[V(T)^{2} X_{N}^{2}\right]$ has a finite limit. But

$$
E\left[V(T)^{2} X_{N}^{2}\right]=e^{2 \mu_{w} T+\sigma_{w}^{2} T} E\left[e^{2 \bar{\sigma}^{\top} W(T)-2 \sigma_{w}^{2} T} X_{N}^{2}\right] .
$$

By Girsanov's theorem, the expectation on the right is the second moment of $X_{N}$ in a model in which each original asset drift $\mu_{i}$ is changed to $\mu_{i}+2 \sigma_{i}^{\top} \bar{\sigma}$. From Proposition 1 , we have convergence of the second moment of $X_{N}$, regardless of the values of the drifts, as long as they are constants. The last statement in the proposition follows from (14) and part (i).

\section{A.4 Proofs of Theorems 2 and 3}

Before proceeding with the proofs of the theorems, we record the conditional distribution of the Brownian increments in the following lemma:

Lemma 1 Conditional on $\bar{\sigma}^{\top} W(T)=u, u \in \mathbb{R}$, the increments $\Delta W(1), \ldots, \Delta W(N)$ are jointly normal. Each $\Delta W(n)$ has conditional distribution

$$
\left(\Delta W(n) \mid \bar{\sigma}^{\top} W(T)=u\right) \sim N\left(\frac{\bar{\sigma}}{\sigma_{w}^{2} N} u, \Delta t\left(I-\frac{\bar{\sigma} \bar{\sigma}^{\top}}{\sigma_{w}^{2} N}\right)\right),
$$

and each pair of increments $\Delta W(n), \Delta W(m), m \neq n$, has covariance matrix $-\bar{\sigma} \bar{\sigma}^{\top} \Delta t / \sigma_{w}^{2}$. The conditional joint distribution of $\Delta W(1), \ldots, \Delta W(N)$ coincides with the unconditional joint distribution of

$$
\Delta \tilde{W}(n)=\Delta W(n)-\frac{\bar{\sigma} \bar{\sigma}^{\top} W(T)}{\sigma_{w}^{2} N}+\frac{\bar{\sigma} u}{\sigma_{w}^{2} N}, \quad n=1, \ldots, N
$$


Proof of Theorem 2. The first assertion follows from the fact that jointly normal random variables remain jointly normal when conditioned on a linear combination. To derive the conditional means and covariances of the increments, we first note that the joint distribution of $\Delta W(n), \Delta W(m)$, and $\bar{\sigma}^{\top} W(T), m \neq n$, is

$$
\left(\begin{array}{c}
\Delta W(n) \\
\Delta W(m) \\
\bar{\sigma}^{\top} W(T)
\end{array}\right) \sim N\left(0,\left(\begin{array}{ccc}
\Delta t I & 0 & \Delta t \bar{\sigma} \\
0 & \Delta t I & \Delta t \bar{\sigma} \\
\Delta t \bar{\sigma}^{\top} & \Delta t \bar{\sigma}^{\top} & \sigma_{w}^{2} T
\end{array}\right)\right)
$$

Now using a standard identity (as in, e.g., Theorem 2.5.1 of Anderson [2]) for the conditional mean and covariance of a normal vector given the values of some components of the vector (in this case, the last component), we get

$$
\left(\begin{array}{c}
\Delta W(n) \\
\Delta W(m)
\end{array} \mid \bar{\sigma}^{\top} W(T)=u\right) \sim N\left(\frac{u}{\sigma_{w}^{2} N}\left(\begin{array}{c}
\bar{\sigma} \\
\bar{\sigma}
\end{array}\right), \Delta t\left[I-\frac{1}{\sigma_{w}^{2} N}\left(\begin{array}{cc}
\bar{\sigma} \bar{\sigma}^{\top} & \bar{\sigma} \bar{\sigma}^{\top} \\
\bar{\sigma} \bar{\sigma}^{\top} & \bar{\sigma} \bar{\sigma}^{\top}
\end{array}\right)\right]\right) .
$$

The conditional distribution (52) and the conditional covariance of $\Delta W(n)$ and $\Delta W(m)$ can be read from this joint distribution. The last assertion in the lemma follows from direct verification that each pair of vectors in (53) has the joint distribution in (54).

Proof of Theorem 2. In light of Lemma 1, we can represent the conditional distributions

$$
\left(\frac{\hat{V}_{N}}{V(T)} \mid V(T)=y_{N}\right)=\left(\prod_{n=1}^{N} \frac{\hat{R}_{n}}{R_{n}} \mid V(T)=y_{N}\right)
$$

as

$$
\prod_{n=1}^{N} \sum_{i=1}^{d} w_{i} \exp \left(\left(\mu_{i}-\mu_{w}\right) \Delta t+\left(\sigma_{w}^{2}-\left\|\sigma_{i}\right\|^{2}\right) \Delta t / 2+\left(\sigma_{i}-\bar{\sigma}\right)^{\top} \Delta \tilde{W}(n)\right),
$$

with $\Delta \tilde{W}(n)$ as in (53) and $u=x \sqrt{N}$. Any limit of (56) is then a weak limit of (55).

We will expand the factors in (56) by following steps similar to those used for $g_{N, n}$ in (37). For each factor in (56), replace $\tilde{W}(n)$ with its definition in (53) (with $u$ in (53) replaced by $x \sqrt{N}$ ), and then replace each $\Delta W(n)$ with $Z_{N, n} \sqrt{\Delta t}$, where $Z_{N, 1}, \ldots, Z_{N, n}$ are independent standard normal vectors in $\mathbb{R}^{d}$. Just as with $g_{N, n}$ in (37), this allows us to write each factor in (56) as a function $G_{N, n}(\sqrt{\Delta})$. Next, we carry out a Taylor expansion of $G_{N, n}$. We find that $G_{N, n}(0)=1, G_{N, n}^{\prime}(0)$, and $G_{N, n}^{\prime \prime}(0) \Delta t / 2=Y_{N, n}+(a / N)$, where

$$
Y_{N, n}=\sum_{i=1}^{d} w_{i}\left(\sigma_{w}^{2}-\left\|\sigma_{i}\right\|^{2}+\left[\left(\sigma_{i}-\bar{\sigma}\right)^{\top} Z_{N, n}\right]^{2} \frac{\Delta t}{2}\right)+\sum_{i=1}^{d} w_{i}\left(\sigma_{i}-\bar{\sigma}\right)^{\top} Z_{N, n} \sqrt{\Delta t}\left(\sigma_{i}-\bar{\sigma}\right)^{\top} \frac{x}{\sigma_{w}^{2} \sqrt{N}}
$$

and

$$
a=\frac{1}{2} \sum_{i=1}^{d} w_{i}\left[\left(\sigma_{i}-\bar{\sigma}\right)^{\top} \bar{\sigma}\right]^{2} \frac{x^{2}}{\sigma_{w}^{4}}
$$


Thus,

$$
\sum_{i=1}^{d} w_{i} \exp \left(\left(\mu_{i}-\mu_{w}\right) \Delta t+\left(\sigma_{w}^{2}-\left\|\sigma_{i}\right\|^{2}\right) \Delta t / 2+\left(\sigma_{i}-\bar{\sigma}\right)^{\top} \Delta \tilde{W}(n)\right)=1+Y_{N, n}+\frac{a}{N}+r_{N, n}
$$

where the remainder $r_{N, n}$ is $O\left(\Delta t^{3 / 2}\right)$. More precisely, each $r_{N, n}$ is the product of a cubic function of normal random vectors and $\xi_{N, n}^{3}$ for some $\xi_{N, n} \in[0, \sqrt{\Delta t}]$. We write the logarithm of (56) as

$$
\sum_{n=1}^{N} \log \left(1+Y_{N, n}+\frac{a}{N}+r_{N, n}\right)
$$

We claim that this sum converges to $a$ in probability.

To justify this claim, we first observe that $E\left[Y_{N, n}\right]=0$ (see (5)) and, by properties of the quadratic variation of Brownian motion,

$$
\sum_{n=1}^{N} Y_{N, n} \quad \text { and } \quad \sum_{n=1}^{N} Y_{N, n}^{2}
$$

both converge to zero in probability. (The first assertion follows from Theorem 1.5.8 of Karatzas and Shreve [26] and the second from their Problem 1.5.11, for which they provide a solution on p.44. These statements can also be verified using the $Z_{N, n}$ rather than the Brownian increments.) By the properties of the remainders noted above, we also have that

$$
\sum_{n=1}^{N} r_{N, n}, \quad \sum_{n=1}^{N} r_{N, n}^{2}, \quad \text { and } \quad \sum_{n=1}^{N} Y_{N, n} r_{N, n}
$$

all converge to zero in probability. Thus,

$$
\sum_{n=1}^{N}\left(Y_{N, n}+\frac{a}{N}+r_{N, n}\right)
$$

converges to $a$ in probability, and

$$
\sum_{n=1}^{N}\left(Y_{N, n}+\frac{a}{N}+r_{N, n}\right)^{2} \quad \text { and } \max _{n=1, \ldots, N}\left|Y_{N, n}+\frac{a}{N}+r_{N, n}\right|
$$

converge to zero in probability. But, for all sufficiently small $v$,

$$
v-v^{2} \leq \log (1+v) \leq v+v^{2},
$$

so (57) converges to $a$ in probability. It follows that (56) converges in probability to $e^{a}$ and thus that (55) converges in distribution to the same constant.

It remains to evaluate $a$. From the definition of $a$, we can write $a=\beta_{L} x^{2} / 2$ with

$$
\beta_{L}=\sum_{i=1}^{d} w_{i}\left[\left(\sigma_{i}-\bar{\sigma}\right)^{\top} \bar{\sigma}\right]^{2} / \sigma_{w}^{4}
$$


But

$$
\begin{aligned}
\sum_{i=1}^{d} w_{i}\left[\left(\sigma_{i}-\bar{\sigma}\right)^{\top} \bar{\sigma}\right]^{2} & =\sum_{i=1}^{d} w_{i}\left[\left(\sigma_{i}^{\top} \bar{\sigma}\right)^{2}-2 \sigma_{i}^{\top} \bar{\sigma} \sigma_{w}^{2}+\sigma_{w}^{4}\right] \\
& =\sum_{i=1}^{d} w_{i}\left[\left(\sigma_{i}^{\top} \bar{\sigma}\right)^{2}-\sigma_{w}^{4}\right]=\bar{\sigma}^{\top} B \bar{\sigma}
\end{aligned}
$$

using (13). Repeating the steps from (45) to (46) establishes the second expression for $\beta_{L}$ in the statement of the theorem.

Proof of Theorem 3: Using the conditional distribution of $\Delta W(n)$ in (52), we get

$$
\begin{aligned}
E\left[\Delta W(n)^{\top} B \Delta W(n) \mid \bar{\sigma}^{\top} W(T)=x\right] & =\frac{\bar{\sigma}^{\top} B \bar{\sigma}}{N^{2} \sigma_{w}^{4}} x^{2}+\operatorname{Tr}\left(B \cdot \Delta t\left(I-\frac{\bar{\sigma} \bar{\sigma}^{\top}}{N \sigma_{w}^{2}}\right)\right) \\
& =\frac{\bar{\sigma}^{\top} B \bar{\sigma}}{N^{2} \sigma_{w}^{4}} x^{2}+\operatorname{Tr}(B) \Delta t-\frac{\bar{\sigma}^{\top} B \bar{\sigma}}{N^{2} \sigma_{w}^{2}} T .
\end{aligned}
$$

From (11), we therefore get

$$
E\left[\epsilon_{n} \mid \bar{\sigma}^{\top} W(T)=x\right]=\frac{1}{2}\left(\frac{\bar{\sigma}^{\top} B \bar{\sigma}}{N^{2} \sigma_{w}^{4}} x^{2}-\frac{\bar{\sigma}^{\top} B \bar{\sigma}}{N^{2} \sigma_{w}^{2}} T\right)=\frac{\bar{\sigma}^{\top} B \bar{\sigma}}{2 \sigma_{w}^{4}}\left(\frac{x^{2}-\sigma_{w}^{2} T}{N^{2}}\right),
$$

and (21) follows using the first expression for $\beta_{L}$ in (19).

\section{A.5 Proof of Proposition 5}

The first $K$ terms in (31) are independent of each other; the remaining $K(K-1) / 2$ terms are uncorrelated with each other and with the first $K$ terms. Thus, the variance of (31) is the sum of the variances of the individual terms. For each of the first $K$ terms, we have

$$
\operatorname{Var}\left[\frac{1}{2} \Delta W_{k}^{\top} B \Delta W_{k}\right]=\frac{1}{4}\left(\frac{\Delta t}{K}\right)^{2} \operatorname{Var}\left[Z^{\top} B Z\right]=\frac{\Delta t^{2}}{2 K^{2}} \operatorname{Tr}\left(B^{2}\right),
$$

where $Z \sim N(0, I)$ in $\mathbb{R}^{d}$; and, for each of the remaining terms, we have

$$
\operatorname{Var}\left[\frac{1}{2} \Delta W_{j}^{\top} C \Delta W_{k}\right]=\frac{1}{4}\left(\frac{\Delta t}{K}\right)^{2} \operatorname{Var}\left[Z^{\top} C \tilde{Z}\right]=\frac{\Delta t^{2}}{4 K^{2}} \cdot \operatorname{Tr}\left(C^{2}\right),
$$

with $\tilde{Z} \sim N(0, I)$, independent of $Z$. Summing $K$ times the first expression and $K(K-1) / 2$ times the second yields (33).

For (34), we need to evaluate $\operatorname{Tr}\left(C^{2}\right)$. Using the second expression in (30), we get

$$
\operatorname{Tr}\left(C^{2}\right)=\operatorname{Tr}\left(\pi_{1}^{2}\left(\bar{\sigma}_{1} \bar{\sigma}_{1}^{\top}-A_{1}\right)^{2}+B^{2}+2 \pi_{1} B\left(\bar{\sigma}_{1} \bar{\sigma}_{1}^{\top}-A_{1}\right)\right) .
$$

Then,

$$
\operatorname{Tr}\left(\left(\bar{\sigma}_{1} \bar{\sigma}_{1}^{\top}-A_{1}\right)^{2}\right)=2 \sigma_{L}^{2}(1)
$$

$\operatorname{Tr}\left(B^{2}\right)=2 \sigma_{L}^{2}$, and

$$
B\left(\bar{\sigma}_{1} \bar{\sigma}_{1}^{\top}-A_{1}\right)=\sigma_{L}^{2}(1, \cdot) .
$$

The details of these calculations are similar to the steps in (44)-(47) and are therefore omitted. 


\section{References}

[1] Artzner, P., Delbaen, F., Eber, J.-M., and Heath, D. (1999) Coherent measures of risk, Mathematical Finance 9, 203-228.

[2] Anderson, T.W. (1984) An Introduction to Multivariate Statistical Analysis, Second Edition, Wiley, New York.

[3] Bangia, A., Diebold, F., Schuermann, T., and Stroughair, J. (1998) Modeling liquidity risk, with implications for traditional market risk measurement and management, Working Paper 99-06, Wharton School Center for Financial Institutions, University of Pennsylvania.

[4] Basel Committee on Banking Supervision (2007) Guidelines for computing capital for incremental default risk in the trading book (BCBS 134), Bank for International Settlements, Basel, Switzerland. Available at www.bis.org.

[5] Basel Committee on Banking Supervision (2009) Guidelines for computing capital for incremental risk in the trading book (BCBS 149), Bank for International Settlements, Basel, Switzerland. Available at www.bis.org.

[6] Bertsimas, D., Kogan, L., and Lo, A.W. (2000) When is time continuous? Journal of Financial Economics 55, 173-204.

[7] Billingsley, P. (1968) Convergence of Probability Measures, Wiley, New York.

[8] Boyle, P., and Emanuel, D. (1980) Discretely adjusted option hedges, Journal of Financial Economics 8, 259-282.

[9] Broadie, M., Glasserman, P., and Kou, S.-G. (1997) A continuity correction for discrete barrier options, Mathematical Finance 7, 325-348.

[10] Chung, K.-L. (1974) A Course in Probability Theory, 2nd Edition, Academic Press, New York.

[11] Crouhy, M., Galai, D., and Mark, R. (2000) Risk Management, McGraw-Hill, New York.

[12] Detemple, J., Garcia, R., and Rindisbacher, M. (2006) Asymptotic properties of Monte Carlo estimators of diffusion processes, Journal of Econometrics 134, 1-68.

[13] Duan, J.C. (1997) Augmented GARCH(p,q) process and its diffusion limit, Journal of Econometrics $79,97-127$.

[14] Dubil, R. (2003) How to include liquidity in a market VaR statistic?, Journal of Applied Finance, 13(1), 19-28. 
[15] Duffie, D., and Sun, T.-S. (1990) Transactions costs and portfolio choice in a discretecontinuous-time setting, Journal of Economic Dynamics and Control 14, 35-51.

[16] Dufresne, D. (2004) The log-normal approximation in financial and other computations, Advances in Applied Probability 36, 747-773.

[17] Duncan, E., Portilla, A., Seal, K., and Scott, S. (2009) Joint Association response to "Revisions to the Basel II market risk framework" (BCBS 148) and "Guidelines for computing capital for incremental risk in the trading book" (BCBS 149), International Swaps and Derivatives Association, London. Available at www.isda.org.

[18] Finger, C. (2009) IRC comments, RiskMetrics Group Research Monthly (February 2009), www.riskmetrics.com.

[19] Gordy, M.B., and Howells, B. (2006) Procyclicality in Basel II: Can we treat the disease without killing the patient?, Journal of Financial Intermediation 15, 395-417.

[20] Guasoni, P., Huberman, G., and Wang, Z. (2007) Performance maximization of actively managed funds, Working paper, Federal Reserve Bank of New York.

[21] Haug, E. G. (2007) Derivatives Models on Models, Wiley, New York.

[22] Hörfelt, P. (2003) Extension of the corrected barrier approximation by Broadie, Glasserman, and Kou, Finance and Stochastics 7, 231-243.

[23] Howison, S., and Steinberg, M. (2007) A matched asymptotic expansions approach to continuity corrections for discretely sampled options. I. Barrier options, Applied Mathematical Finance 14, 63-89.

[24] Jacod, J., and Protter, P. (1998) Asymptotic error distributions for the Euler method for stochastic differential equations, Annals of Probability 26, 267-307.

[25] Jorion, P. (2006) Value at Risk, Third Edition, McGraw-Hill, New York.

[26] Karatzas, I., and Shreve, S. (1991) Brownian Motion and Stochastic Calculus, Second Edition, Springer-Verlag, New York.

[27] Kurtz, T.G., and Protter, P. (1991) Wong-Zakai corrections, random evolutions and numerical schemes for SDEs, in Stochastic Analysis, Academic Press, New York, 331-346.

[28] Lai, T.L., Yao, Y.C., and AitSahalia, F. (2007) Corrected random walk approximations to free boundary problems in optimal stopping, Advances in Applied Probability 39, 753-775. 
[29] Leland, H.E. (1985) Option pricing and replication with transactions costs, Journal of Finance 40, 1283-1301.

[30] McNeil, A., Frey, R., and Embrechts, P. (2005) Quantitative Risk Management: Concepts, Techniques, and Tools, Princeton University Press, Princeton, New Jersey.

[31] Morton, A., and Pliska, S. (1995) Optimal portfolio management with fixed transaction costs, Mathematical Finance 5, 337-356.

[32] Rockafellar, R.T., and Uryasev, S. (2000) Optimization of conditional value-at-risk, Journal of Risk 2, 21-41.

[33] Sebton, E., Epperlein, E., Smillie, A., Picoult, E., Farrall, S., Kwiatkowski, J., Skaane, H., Wilde, T., Kausch, H., and Cooper, S. (2007) Industry technical paper on incremental default risk, International Swaps and Derivatives Association, London. Available at www.isda.org.

[34] Smillie, A., and Epperlein, E. (2007) Compliance by default, Risk 20(December):96-100. 